\title{
Regulation of terpenoid biosynthesis by miRNA in Persicaria minor induced by Fusarium oxysporum
}

\author{
Abdul Fatah A. Samad ${ }^{1,5}$, Reyhaneh Rahnamaie-Tajadod², Muhammad Sajad ${ }^{2,4}$, Jaeyres Jani ${ }^{3}$,
} Abdul Munir Abdul Murad', Normah Mohd Noor ${ }^{2}$ and Ismanizan Ismail ${ }^{1,2^{*}}$

\begin{abstract}
Background: Persicaria minor (kesum) is an herbaceous plant with a high level of secondary metabolite compounds, particularly terpenoids. These terpenoid compounds have well-established roles in the pharmaceutical and food industries. Although the terpenoids of $P$. minor have been studied thoroughly, the involvement of microRNA (miRNA) in terpenoid regulation remains poorly understood and needs to be explored. In this study, $P$. minor plants were inoculated with the pathogenic fungus Fusarium oxysporum for terpenoid induction.

Result: SPME GC-MS analysis showed the highest terpenoid accumulation on the 6th day post-inoculation (dpi) compared to the other treatment time points ( $0 \mathrm{dpi}, 3 \mathrm{dpi}$, and $9 \mathrm{dpi}$ ). Among the increased terpenoid compounds, a-cedrene, valencene and $\beta$-bisabolene were prominent. P. minor inoculated for 6 days was selected for miRNA library construction using next generation sequencing. Differential gene expression analysis showed that 58 miRNAs belonging to 30 families had significantly altered regulation. Among these 58 differentially expressed genes (DEGs), 33 miRNAs were upregulated, whereas 25 miRNAs were downregulated. Two putative novel pre-miRNAs were identified and validated through reverse transcriptase PCR. Prediction of target transcripts potentially involved in the mevalonate pathway (MVA) was carried out by psRobot software, resulting in four miRNAs: pmi-miR530, pmimiR6173, pmi-miR6300 and a novel miRNA, pmi-Nov_13. In addition, two miRNAs, miR396a and miR398f/g, were predicted to have their target transcripts in the non-mevalonate pathway (MEP). In addition, a novel miRNA, pmiNov_12, was identified to have a target gene involved in green leaf volatile (GLV) biosynthesis. RT-qPCR analysis showed that pmi-miR6173, pmi-miR6300 and pmi-nov_13 were downregulated, while miR396a and miR398f/g were upregulated. Pmi-miR530 showed upregulation at $9 \mathrm{dpi}$, and dynamic expression was observed for pmi-nov_12. Pmi6300 and pmi-miR396a cleavage sites were detected through degradome sequence analysis. Furthermore, the relationship between miRNA metabolites and mRNA metabolites was validated using correlation analysis.
\end{abstract}

Conclusion: Our findings suggest that six studied miRNAs post-transcriptionally regulate terpenoid biosynthesis in $P$. minor. This regulatory behaviour of miRNAs has potential as a genetic tool to regulate terpenoid biosynthesis in $P$. minor.

Keywords: Terpenoid biosynthesis, miRNA, P. minor, F. Oxysporum, Deep sequencing, Post-transcriptional regulation

\footnotetext{
* Correspondence: maniz@ukm.edu.my

${ }^{1}$ School of Biosciences and Biotechnology, Faculty of Science and

Technology, Universiti Kebangsaan Malaysia, UKM, 43600 Bangi, Selangor,

Malaysia

${ }^{2}$ Institute of Systems Biology, Universiti Kebangsaan Malaysia, UKM, 43600

Bangi, Selangor, Malaysia

Full list of author information is available at the end of the article
}

(c) The Author(s). 2019 Open Access This article is distributed under the terms of the Creative Commons Attribution 4.0 International License (http://creativecommons.org/licenses/by/4.0/), which permits unrestricted use, distribution, and reproduction in any medium, provided you give appropriate credit to the original author(s) and the source, provide a link to the Creative Commons license, and indicate if changes were made. The Creative Commons Public Domain Dedication waiver (http://creativecommons.org/publicdomain/zero/1.0/) applies to the data made available in this article, unless otherwise stated. 


\section{Background}

Humans exploit the secondary metabolites from medicinal plants in the form of flavouring agents, perfumes, insecticides, dyes and drugs, among other products. Previous studies have shown that more than 100,000 phytochemicals have been isolated from different plant sources [1]. According to the British Nutrition Foundation, secondary metabolites (SMs) are divided into four major classes: terpenoids (volatile compounds, carotenoids and glycosides), phenolic compounds (phenolic acids, tannins and flavonoids), nitrogen-containing compounds (cyanogenic glucosides and alkaloids) and sulfur-containing compounds (thionine, defensin and lectin) [2]. The terpenoid classes consist of volatile and non-volatile compounds. Though these compounds exist in complex structures, they all are made up of isoprene units (C5). Most volatile terpenoids are monoterpenes $(\mathrm{C} 10)$ and sesquiterpenes $(\mathrm{C} 15)$, while non-volatile terpenoids include diterpenes (C20), triterpenes (C30) and tetraterpenes (C40) [3]. Terpenoids are widely used in pharmaceuticals, cosmetic fragrances and the food industry [4]. To date, more than 40,000 known terpenoid compounds have been identified, mostly from plants [5].

Persicaria minor (locally known as kesum) is an aromatic plant that is widespread in Southeast Asia, especially in Malaysia, Thailand, Laos, Indonesia and Vietnam. This plant has been used in dishes and medical purposes for many years. The Malaysian government enlisted P. minor in the National Agro Food Policy to ensure its adequate supply and as a platform to strengthen the agriculturalbased economy [6]. P. minor has also gained attention due to its abundance of secondary metabolites, especially flavonoids and terpenoids, which are used in the food, fragrance and pharmaceutical industries [7]. P. minor leaves produce the highest content of terpenoids compared to other organs [8]. These compounds play important roles in attracting pollinators, plant defence, and interactions with unfavourable environments. Two different pathways of terpenoid biosynthesis have been identified: the mevalonate (MVA) and non-mevalonate, or methylerythritol 4-phosphate (MEP), pathways [9]. These pathways operate in the cytoplasm and plastid, respectively [9].

Past achievements in unlocking the genes and enzymes involved in each of these pathways has opened up new possibilities for the assessment of terpenoid biosynthesis [10]. At the molecular level, molecules such as DNA, mRNA, transcription factors, and non-coding RNA play roles in regulating the genes involved in these pathways. In addition, these molecules may interact with each other, which can affect the target gene, thus influencing the production of the secondary metabolite [11]. However, the level of secondary metabolites in plants is relatively low because their production depends on plant species, environmental factors, and nutritional sources
$[12,13]$. In addition, the type of secondary metabolites produced by the plant is stimulus-dependent. For example, in the majority of cases, terpenoid compounds are produced in high abundance under biotic stress compared to abiotic [14]. Hence, the elicitation technique was introduced to enhance secondary metabolite production in plants using a biotic elicitor [13, 15]. Elicitation using fungi, especially $F$. oxysporum, has been reported to enhance terpenoid contents in plants [16-19].

To understand the changes at the molecular level, the relationship between miRNA and mRNA needs to be explored, because the interaction between these two RNA species may play a significant role in plant secondary metabolite production. miRNAs are a group of noncoding RNAs with small sizes $(\sim 20 \mathrm{bp})$ that act as a gene regulators by negatively regulating mRNAs via cleavage or translational inhibition [11, 20]. The first miRNA, lin-4 miRNA, was discovered in C. elegans through forward genetic analysis. Later, several methods were developed to discover these tiny RNAs, such as cloning and computational methods [20]. However, these methods had their own limitations. Due to the ability of several miRNAs to target single mRNAs, using forward genetic techniques for miRNA discovery is very difficult unless the miRNA of interest has only one target $[11,20]$. The cloning method is also limited to highly expressed miRNAs [20]. Computational approaches could be useful for miRNA discovery but have limitations in detecting novel miRNAs in other species, since these approaches are based on homology searches. In addition, computational approaches still require experimental validation [20]. Next generation sequencing (NGS), a more recent approach, has also been introduced for miRNA study, and this method has been found to be more advanced and efficient. This approach can detect low copy number miRNAs at levels of one transcript per million [21, 22]. To date, a total of 38,589 miRNAs from animals, plants, and viruses have been discovered and deposited in public miRNA databases [23]. These miRNAs have the ability to fine-tune many biological and physiological processes such as plant development and stress response by regulating a number of target genes in a time-sensitive manner [11].

Interestingly, miRNAs were reported to be involved in plant secondary metabolite regulation, such as terpenoid, phenolic and nitrogen-containing compound biosynthesis [1]. These interactions could hold potential for future genetic manipulation. In addition, using miRNA as a tool for genetic engineering could be highly beneficial because single miRNAs can target more than single mRNAs and vice versa. Authenticated in silico miRNA discovery and wet lab approaches for miRNA target validation are required prior to their application to plant genetic systems. Thus, in this study, the regulatory roles of miRNAs in the terpenoid biosynthesis pathway were 
explored in $P$. minor induced by $F$. oxysporum. Metabolite profiling was carried out for the $F$. oxysporum-inoculated plants to determine the changes in the accumulation of terpenoid compounds. In addition, small RNA libraries were constructed, which led to the characterization and analysis of miRNAs involved in the terpenoid biosynthesis pathway.

\section{Results and discussion}

Testing of $F$. oxysporum inoculation and terpenoid profiling in $P$. minor by SPME-GCMS

To the best of our knowledge, no work on $F$. oxysporum inoculation to induce terpenoid contents has been reported to date in $P$. minor. Therefore, we carried out an inoculation test to determine the elicitation ability of this fungus to increase terpenoid content in P. minor. Comparison between mock-inoculated (C) and Fusariumtreated (F) plants showed different leaf morphologies over the time point of the treatment (Fig. 1). From the beginning of the treatment $(0 \mathrm{dpi})$ to $3 \mathrm{dpi}$, there were no morphological changes observed in either of the $C$ or F samples. At $6 \mathrm{dpi}$, no change was observed in the $\mathrm{C}$ samples, while the $\mathrm{F}$ samples showed wilting symptoms beginning at the tip of the leaf. At $9 \mathrm{dpi}$, the wilting spread to the middle of the leaves in the F samples. At $12 \mathrm{dpi}$, the wilting spread to the whole leaf, which led to the death of the plant. In comparison, no changes were observed in the $C$ samples until the end point, $12 \mathrm{dpi}$. A previous report mentioned an accumulation of terpenoids when plants were attacked by pathogens [24]. Based on Fig. 1, the first symptom appeared at 6 dpi. Theoretically, the test suggested the highest terpenoid content at $6 \mathrm{dpi}$ in $P$. minor inoculated with $F$. oxysporum. However, the SPME-GCMS result was carried out to reveal the terpenoid content at each time point. The initial SPME-GCMS data are provided in Additional file 1. The heatmap generated (Fig. 2) indicated a significant increase in three volatile compounds: $\alpha$-cedrene (1H-3a,7-methanoazulene, 2,3,4,7,8,8a-hexahydro-3,6,8,8-

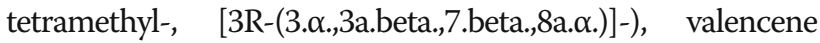
(naphthalene, 1,2,3,5,6,7,8,8a-octahydro-1,8a-dimethyl-7-(1-

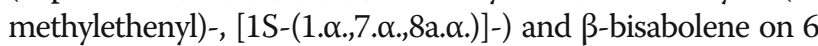
dpi. Hence, samples at the 6 dpi time point were selected for small RNA library construction.

\section{High-throughput small RNA sequencing analysis}

Small RNA sequencing for the $\mathrm{C}$ and $\mathrm{F}$ libraries generated approximately $12,409,685$ and $42,985,084$ reads, respectively. The average numbers and proportions of the different categories are presented in Table 1. Trimming of adaptor index sequences was carried out, and lowquality reads were removed to produce clean reads. Reads with lengths between $18 \mathrm{nt}$ and $30 \mathrm{nt}$ were used for annotation, while the rest were discarded. In total, approximately 7,724,932 and 25,726,524 unique reads were produced for the $\mathrm{C}$ and $\mathrm{F}$ libraries, respectively. The length distribution of the small RNAs in the $C$ and F libraries is shown in Fig. 3. The most abundant small RNAs were $22 \mathrm{nt}$ in length, followed by $20 \mathrm{nt}$, which belonged to the F library. Previous studies have reported that more than $60 \%$ of all plant miRNAs are $21 \mathrm{nt}$ in length [25]. The clean reads from each $P$. minor library were annotated according to the miRBase version 21

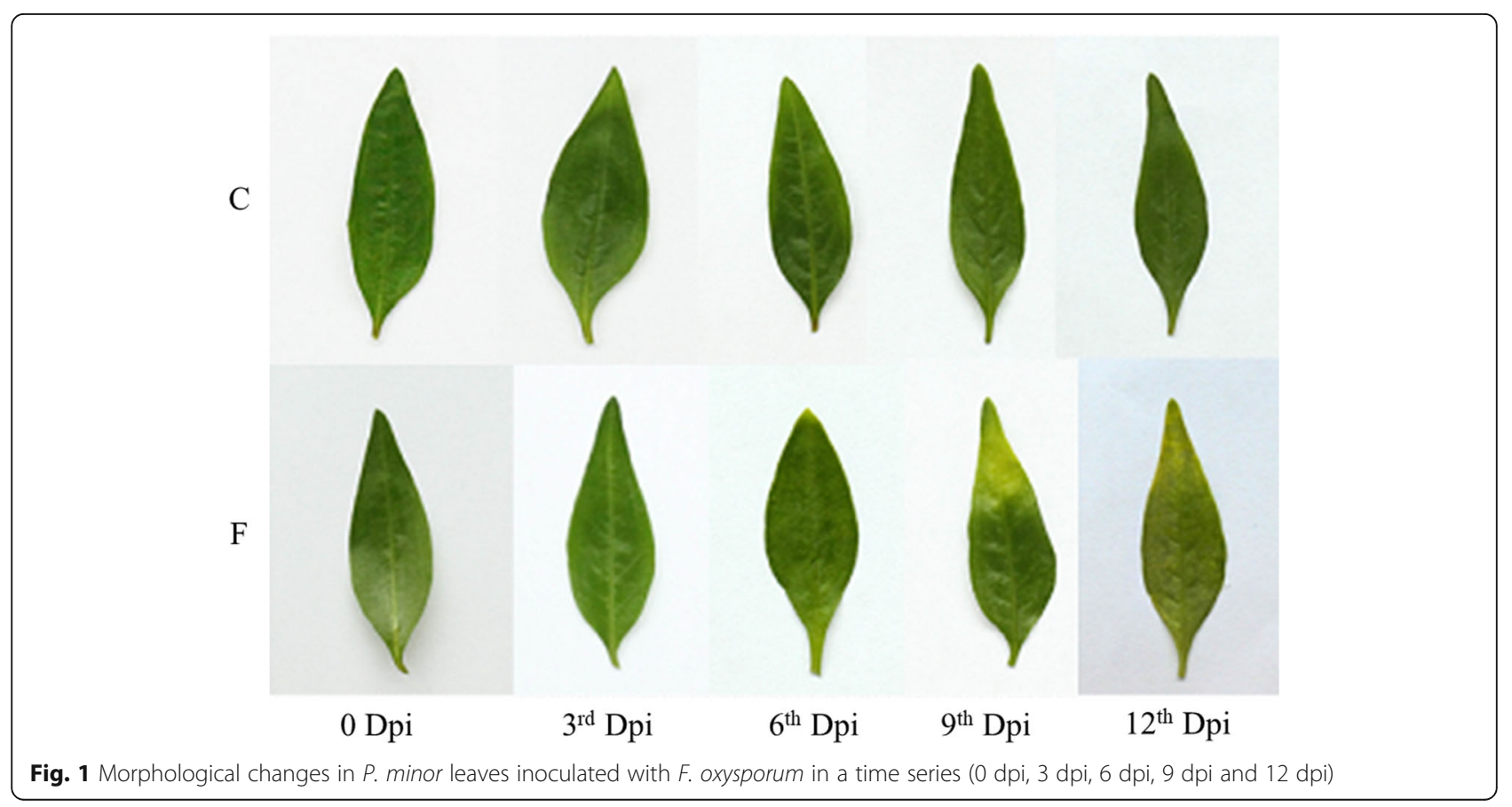




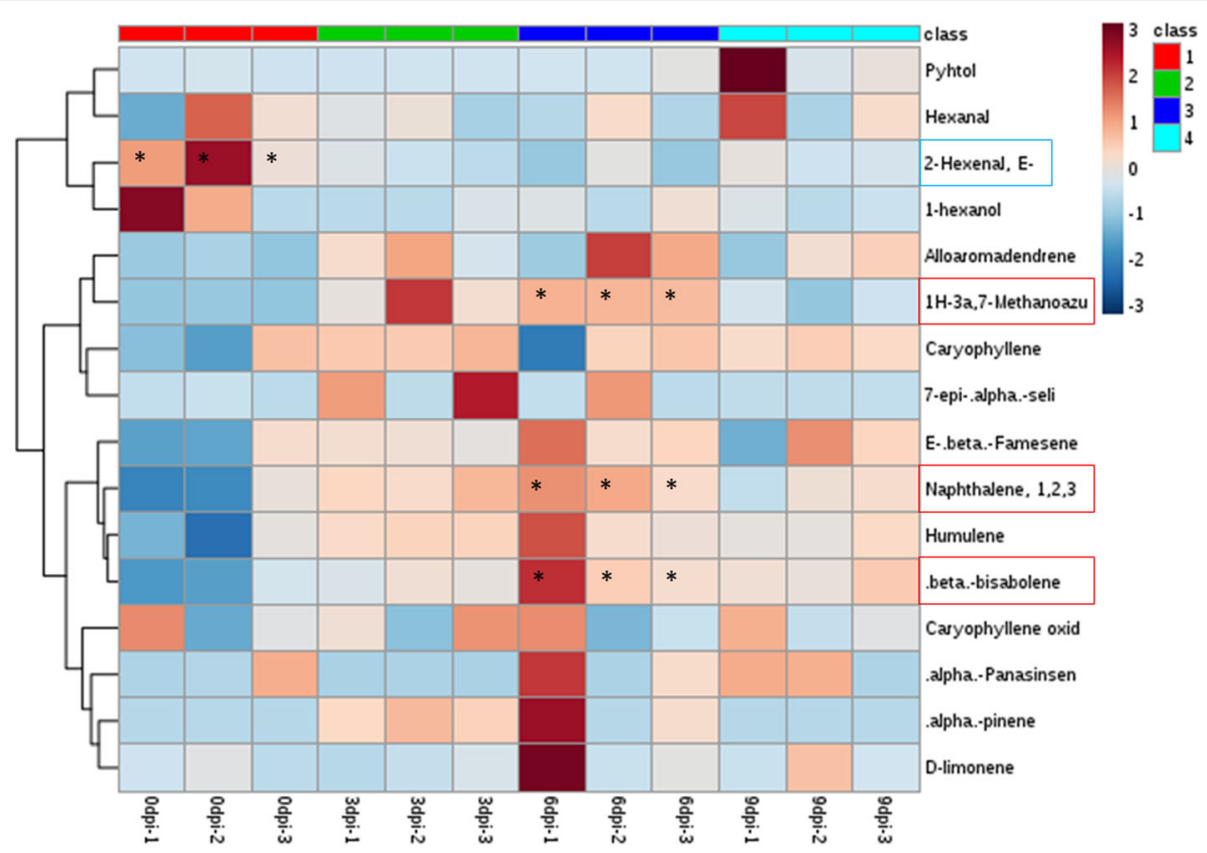

Fig. 2 Heat map representing changes in relative metabolite contents of Fusarium-inoculated and control plants detected by SPME GC-MS experiments. Asterisks indicate statistically significant differences $(P<0.05)$ among all time points by ANOVA and Fisher's LSD test. The compounds in the blue and red boxes represent the statistically significant GLV and terpenoids, respectively

and Rfam databases. From the total clean reads, the C library generated $111,235 \quad(1.44 \%)$ miRNA sequences, which represented $2,610(0.18 \%)$ unique reads. On the other hand, the $\mathrm{F}$ library generated $711,498(2.77 \%)$ miRNA sequences, representing 6 , $131(0.18 \%)$ unique reads. Mapping with Rfam resulted in 15.48 and $18.13 \%$ sequences in the $\mathrm{C}$ and $\mathrm{F}$ libraries, respectively, belonging to non-coding RNAs other than miRNA. The rest of the sequences were marked as unannotated sequences in both libraries.
Differential expression of miRNAs in $\mathrm{C}$ and $\mathrm{F}$ libraries

The overall expression of miRNAs in both libraries is shown in the volcano plot (Fig. 4). The plot showed significant changes in the regulation of 58 miRNAs. Among these significantly regulated miRNAs, 31 were upregulated and 27 were downregulated. Additionally, a heatmap was generated to compare the expression of 58 significantly regulated miRNAs in the $\mathrm{C}$ and $\mathrm{F}$ libraries (Fig. 5). Further details about the significantly regulated miRNAs are documented in Table 2.

Table 1 Average statistic for deep sequencing results in C and F sample

\begin{tabular}{|c|c|c|c|c|}
\hline & Total reads & Percentages (\%) & Unique reads & Percentages (\%) \\
\hline \multicolumn{5}{|l|}{ C } \\
\hline Raw reads & $12409685 \pm 7070024$ & & & \\
\hline Clean reads & $7724932 \pm 4736271$ & 100.00 & $1451635 \pm 830002$ & 100.00 \\
\hline miRNA & $111235 \pm 84067$ & 1.44 & $2610 \pm 1151$ & 0.18 \\
\hline rRNA/tRNA/snoRNA & $1195917 \pm 667460$ & 15.48 & $100356 \pm 35621$ & 6.91 \\
\hline Unannotated & $6417780 \pm 3984743$ & 83.07 & $1348660 \pm 793229$ & 92.91 \\
\hline \multicolumn{5}{|l|}{$\mathrm{F}$} \\
\hline Raw reads & $42985084 \pm 144059$ & & & \\
\hline Clean reads & $25726524 \pm 15406396$ & 100.00 & $3371142 \pm 1880202$ & 100.00 \\
\hline miRNA & $711498 \pm 657406$ & 2.77 & $6131 \pm 2710$ & 0.18 \\
\hline rRNA/tRNA/snoRNA & $4664038 \pm 2660762$ & 18.13 & $182761 \pm 83483$ & 5.42 \\
\hline Unannotated & $20351078 \pm 12088100$ & 79.11 & $3182248 \pm 1794008$ & 94.40 \\
\hline
\end{tabular}




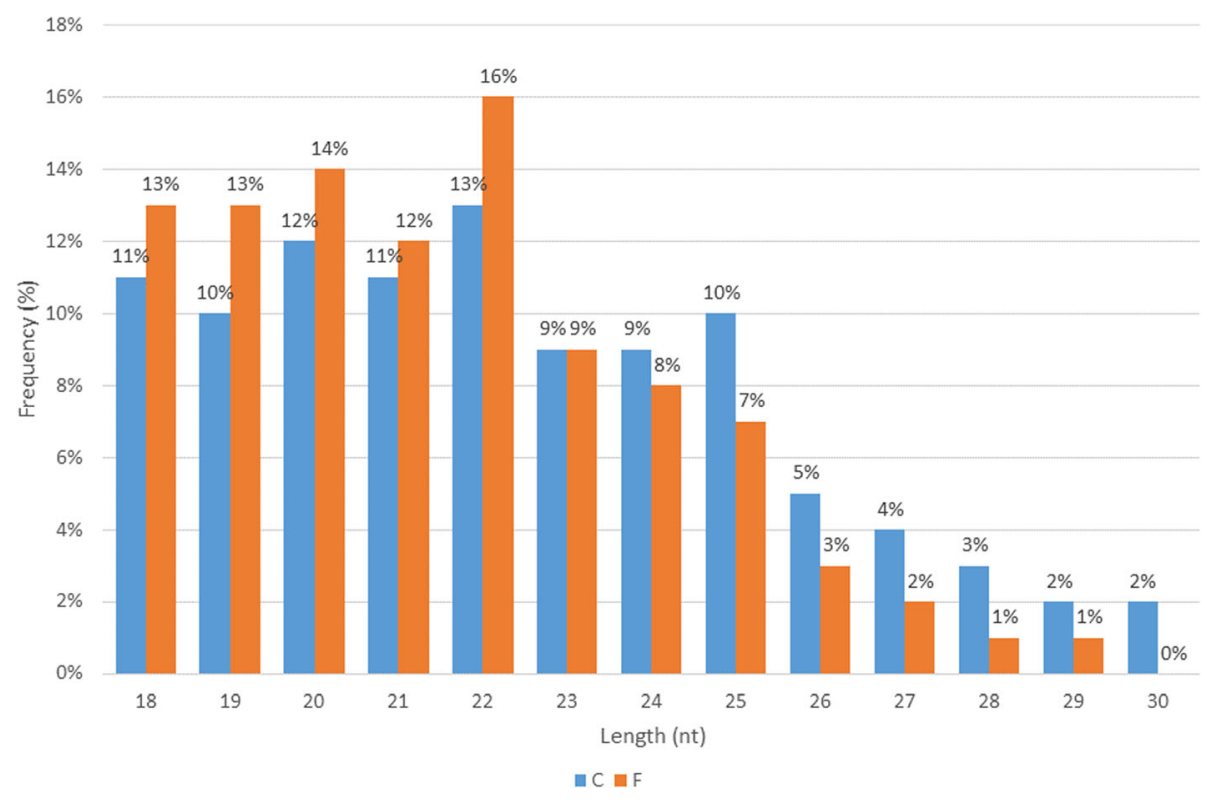

Fig. 3 Length distributions of small RNAs in the $C$ and $F$ libraries

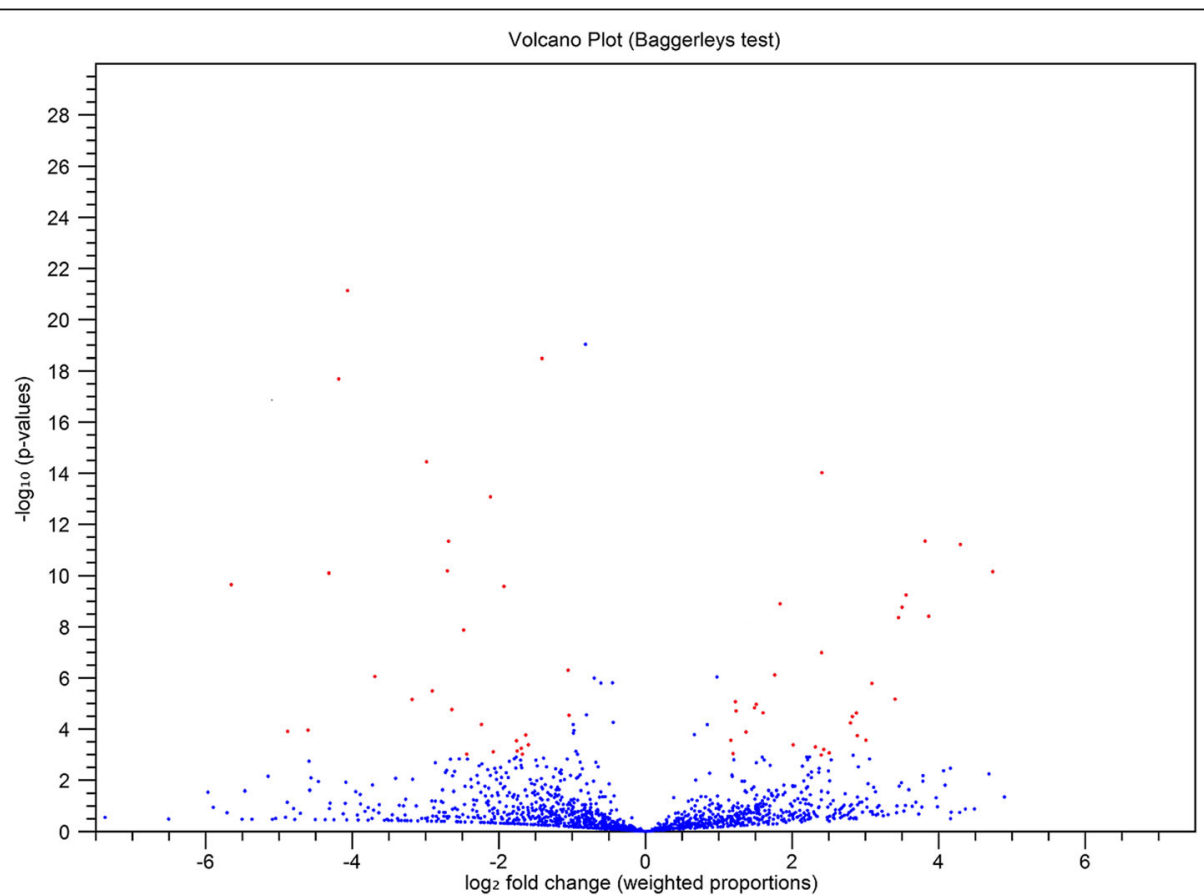

Fig. 4 Volcano plot showing overall miRNA expression. The plot was constructed based on the log 2 fold change on the $x$-axis and -log 10 Pvalues on the $y$-axis. The blue and red dots in the plot represent miRNAs. The blue dots at positive values on the $x$-axis show miRNAs that were not significantly upregulated, whereas red dots at positive values on the $x$-axis showed miRNAs that were significantly upregulated. The blue dots at negative values on the $\mathrm{x}$-axis showed miRNAs that were not significantly downregulated, whereas the red dots at negative values on the $\mathrm{x}$-axis showed miRNAs that were significantly downregulated 


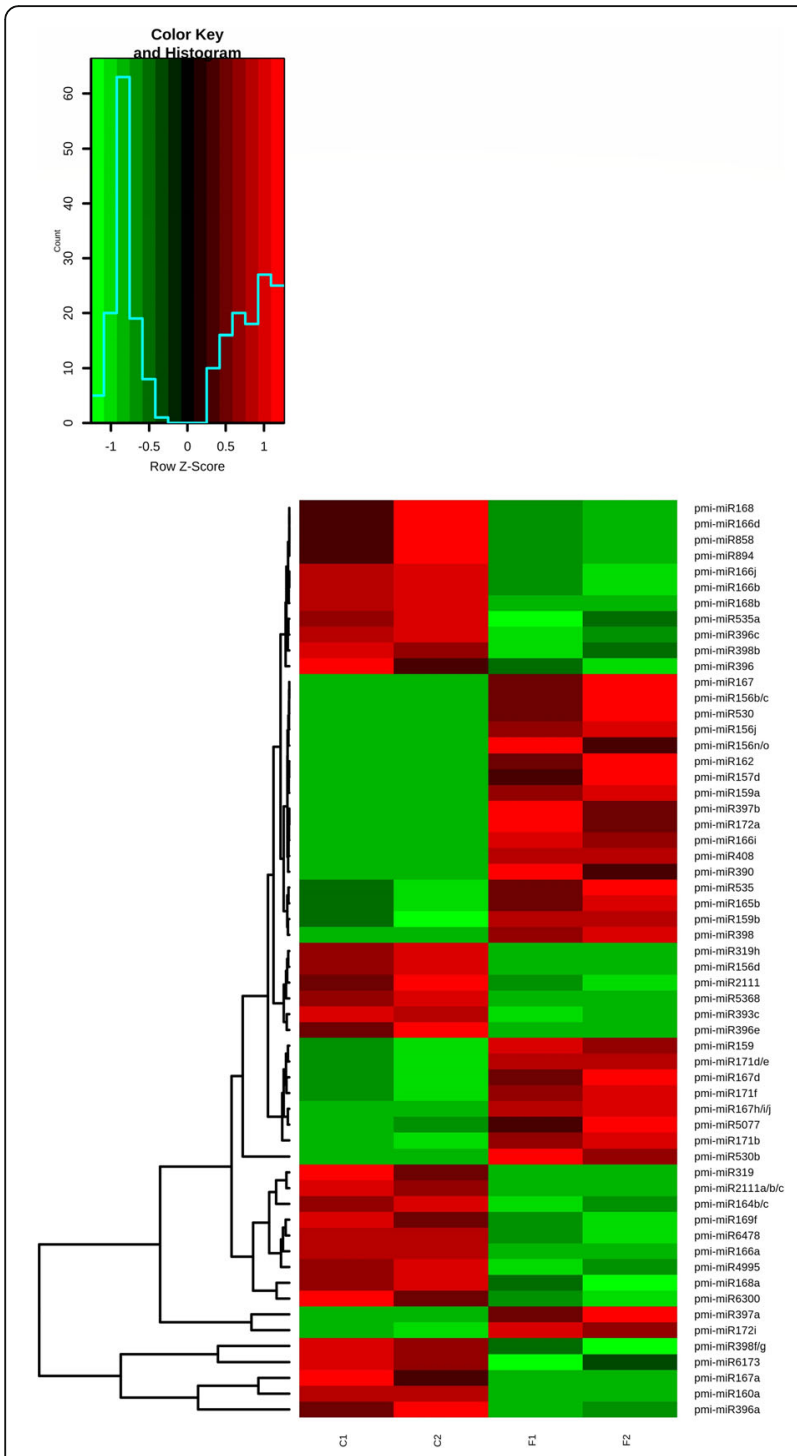

Fig. 5 Heatmap representing miRNAs significantly altered by Baggerley's test $(P<0.05)$ in the $C$ and $F$ libraries Green colour indicates low expression of miRNA, while red colour indicates high expression of miRNA

\section{Common and specific miRNAs}

A Venn diagram was generated to show the common and specific miRNAs in the $\mathrm{C}$ and $\mathrm{F}$ libraries (Fig. 6). Of the 58 miRNAs responsive to $F$. oxysporum treatment, 42 miRNAs were common to both libraries. Two miRNAs, pmi-miR168b and pmi-miR2111, were found to be specific to the $C$ library, whereas 14 miRNAs were specific to the F library.

\section{Discovery of novel miRNAs}

To discover novel miRNA sequences in $P$. minor, all unannotated small RNAs were searched against the $P$. minor transcriptome data under accession number SRX669305 [26]. After searching for hairpin structures (Fig. 7) and performing MFEI calculations (Table 3), two unique sequences were identified as putative novel miRNAs in $P$. minor. These putative novel miRNAs were named pmi-nov_miR12 and pmi-nov_miR13. The secondary structures of both miRNAs were validated through reverse transcriptase PCR (Fig. 8). The sizes of the pmi-nov_miR12 precursor (pre-nov_12) and pminov_miR13 precursor (pre-nov_13) are $\sim 80$ bp and $\sim 75$ $\mathrm{bp}$, respectively. The sizes of the validated precursors were slightly smaller than the predicted sizes. These smaller sizes might be due to the primers, which were designed to avoid the bulge structure in the hairpin structures. The bulge region in the hairpin structure could have reduced the efficiency of primer binding to the DNA template [27].

\section{Prediction and functional annotation of target genes}

In plants, miRNAs are involved in many biological processes by negatively regulating their target genes/transcripts $[11,28]$. In this study, we searched for putative target genes against the $P$. minor transcriptomic library software with mismatch score of 4.0 [29]. This prediction resulted in a total of 111 potential target genes in P. minor. Among them, there were 108 target genes for 58 conserved miRNAs and 3 for two novel miRNAs (Table 4). The majority of the miRNAs have more than one target gene. The target genes were classified according to the WEGO database into cellular component, biological process and molecular function categories (Fig. 9).

\section{Analysis of target transcripts involved in terpenoid pathway}

The terpenoid pathway consists of two distinct pathways, MVA and MEP. From the target prediction (Table 4), a total of seven miRNA targets were discovered that seemed to be directly involved in these terpenoid biosynthesis pathways. In the MVA pathway, the target involved were diphosphomevalonate decarboxylase (MVD), targeted by pmi-miR530; sesquiterpene synthase and farnesyl diphosphate synthase (FDS), targeted by pmi-miR6173; 3-hydroxy-3-methylglutaryl-coenzyme A reductase (HMGR), targeted by pmi-miR6300; and mevalonate kinase (MVK), targeted by pmi-nov_13. In the MEP or non-mevalonate pathway, the identified targets were 1-deoxy-d-xylulose-5-phosphate synthase (DXS) and 1-deoxy-d-xylulose-5-phosphate reductoisomerase (DXR), which were targeted by pmi-miR396a and pmi-miR398f/g, respectively. In addition, two more targets, peroxidase and alcohol dehydrogenase (ADH), were targeted by pmi-miR396a and pmi-nov_12, respectively. Both of these targets were included in this analysis, because peroxidase is involved in the early signalling of secondary metabolite biosynthesis, and ADH is an enzyme involved in green leaf volatile (GLV) biosynthesis. GLV compounds have been reported to have similar 
Table 2 Statistically significant miRNA under inoculation of $F$. oxysporum $(P<0.05)$

\begin{tabular}{|c|c|c|c|}
\hline miRNA & Sequences ( $5^{\prime}$ to $3^{\prime}$ ) & $P$-values & FDR values \\
\hline $\mathrm{pmi}$-miR156b/c & TTGACAGAAGATAGAGAGCACGA & $2.78 \mathrm{E}-4$ & 0.02 \\
\hline pmi-miR156d & ACTCTCTGTGCTTCTGTCATCA & $2.04 \mathrm{E}-18$ & $1.88 \mathrm{E}-15$ \\
\hline pmi-miR156j & TTGACAGAAGAGAGTGAGAA & $1.12 \mathrm{E}-4$ & $7.94 \mathrm{E}-3$ \\
\hline pmi-miR156n/o & TGGCAGAAGAGAGTGAGCACAA & $9.84 \mathrm{E}-4$ & 4.67E-2 \\
\hline pmi-miR157d & CTGACAGAAGATAGAGAGCACGA & $4.12 \mathrm{E}-4$ & $2.28 \mathrm{E}-2$ \\
\hline pmi-miR159 & TTGGATTGGAGGGAGCTCTA & 4.56E-12 & $2.22 \mathrm{E}-9$ \\
\hline pmi-miR159a & CTTGGATTGAAGGGAGCTA & $3.50 \mathrm{E}-6$ & 4.11E-4 \\
\hline pmi-miR159b & TTTGGATTGAAGGGAGCTCTCCA & $5.78 \mathrm{E}-5$ & 4.60E-3 \\
\hline pmi-miR160a & GCGTATGAGGAGCCAAGCATA & 2.97E-120 & $7.50 \mathrm{E}-117$ \\
\hline pmi-miR162 & ACGATAAACCTCTGCATCCAGA & $1.16 \mathrm{E}-5$ & 1.15E-3 \\
\hline pmi-miR164b/c & TGGAGAAGCAGGGCACGTGCA & $3.64 \mathrm{E}-7$ & $6.23 \mathrm{E}-5$ \\
\hline pmi-miR165b & GGAATGTTGTTGGTTCGATGA & $2.36 \mathrm{E}-5$ & $2.11 \mathrm{E}-3$ \\
\hline pmi-miR166a & CTCGGACCAGGCTTCATTCCCA & $1.63 \mathrm{E}-31$ & $2.35 \mathrm{E}-28$ \\
\hline pmi-miR166b & TCGGACCAGGCTTCACTCCCAA & $1.86 \mathrm{E}-7$ & $3.36 \mathrm{E}-5$ \\
\hline pmi-miR166d & TAGGACCAGGCTTCATTCCCTA & $1.63 \mathrm{E}-4$ & 0.01 \\
\hline pmi-miR166i & GGAATGTCGTCTGGTTCA & $1.87 \mathrm{E}-5$ & $1.73 E-3$ \\
\hline pmi-miR166j & TCGGACCAGGCTTCATTCCCATA & $1.86 \mathrm{E}-7$ & $3.36 \mathrm{E}-5$ \\
\hline pmi-miR167 & TGAAGCTGCCAGCATGATCTITA & $2.78 \mathrm{E}-4$ & 0.01 \\
\hline pmi-miR167a & AGATCATCTGGCAGCTTCACCA & $1.23 \mathrm{E}-4$ & $8.60 \mathrm{E}-3$ \\
\hline pmi-miR167d & TGAAGCTGCCAGCATGATCTATTA & $3.84 \mathrm{E}-9$ & $9.47 \mathrm{E}-7$ \\
\hline pmi-miR167h/i/j & TGAAGCTGCCAGCATGATCTTA & $8.54 \mathrm{E}-6$ & $9.08 \mathrm{E}-4$ \\
\hline pmi-miR168 & TCGCTCGGTGCAGGTCGGGAA & $1.63 \mathrm{E}-4$ & 1.06E-2 \\
\hline pmi-miR168a & CCCGCCTTGCATCAACTGAATCA & $4.69 \mathrm{E}-8$ & $1.01 \mathrm{E}-5$ \\
\hline pmi-miR168b & CCCGCTITGCATCAACTGAATA & $1.25 \mathrm{E}-9$ & $3.52 \mathrm{E}-7$ \\
\hline pmi-miR169f & TAGCCAGGGATGACTTGCCGGA & $5.93 \mathrm{E}-7$ & 9.37E-5 \\
\hline pmi-miR171b & TTGAGCCGTGCCAATATCACA & $6.16 \mathrm{E}-12$ & 2.71E-9 \\
\hline pmi-miR171d/e & TTGAGCCGTGCCAATATCACTA & 2.87E-11 & $1.21 \mathrm{E}-8$ \\
\hline pmi-miR171f & TTGAGCCGTGCCAATATCACAA & $1.71 \mathrm{E}-9$ & 4.55E-7 \\
\hline pmi-miR172a & AGAATCTTGATGATGCTGCACTA & $7.68 \mathrm{E}-6$ & $8.25 \mathrm{E}-4$ \\
\hline pmi-miR172i & AGAATCTTGATGATGCTGCATTA & $9.55 \mathrm{E}-15$ & $6.89 \mathrm{E}-12$ \\
\hline pmi-miR2111 & TAATCTGCATCCTGAGGCCCA & $1.77 \mathrm{E}-6$ & $2.33 \mathrm{E}-4$ \\
\hline pmi-miR2111a/b/c & TAATCTGCATCCTGAGGCTAA & $8.75 \mathrm{E}-13$ & $5.53 \mathrm{E}-10$ \\
\hline pmi-miR319 & CTTGGACTGAAGGGAGCTCCCTA & $3.70 \mathrm{E}-7$ & $6.23 \mathrm{E}-5$ \\
\hline pmi-miR319h & CTTGGACTGAAGGGAGCTCCA & $3.56 \mathrm{E}-15$ & $2.76 \mathrm{E}-12$ \\
\hline pmi-miR390 & AAGCTCAGGAGGGATAGCGCCTA & $3.69 \mathrm{E}-4$ & 0.02 \\
\hline pmi-miR393c & TCCAAAGGGATCGCATTGATCCA & 4.61E-12 & $2.22 \mathrm{E}-9$ \\
\hline pmi-miR396 & TTCCACAGCTITCTTGAACTCCA & $5.40 \mathrm{E}-4$ & $2.90 \mathrm{E}-2$ \\
\hline pmi-miR396a & GTTCAATAAAGCTGTGGGAAA & $2.76 \mathrm{E}-4$ & 0.02 \\
\hline pmi-miR396c & GTTCAAGAAAGCTGTGGGATGA & $1.87 \mathrm{E}-6$ & $2.42 \mathrm{E}-4$ \\
\hline pmi-miR396e & TTCCTCAGCTTTCTTGAACTGA & $4.62 \mathrm{E}-7$ & $7.66 \mathrm{E}-5$ \\
\hline pmi-miR397a & TCATTGAGTGCAGCGTTGATAA & $2.32 \mathrm{E}-5$ & $2.10 \mathrm{E}-1$ \\
\hline pmi-miR397b & TCATTGAGTGCAGCGTTGTTGA & $7.68 \mathrm{E}-6$ & $8.26 \mathrm{E}-4$ \\
\hline pmi-miR398 & TGTGTTCTCGGGTCGCCCCTGTA & $1.02 \mathrm{E}-9$ & $3.22 \mathrm{E}-7$ \\
\hline pmi-miR398b & TGAGTTCTCAGGTCGCCCCTA & $1.07 \mathrm{E}-4$ & 7.77E-3 \\
\hline
\end{tabular}


Table 2 Statistically significant miRNA under inoculation of $F$. oxysporum $(P<0.05)$ (Continued)

\begin{tabular}{|c|c|c|c|}
\hline miRNA & Sequences (5' to $\left.3^{\prime}\right)$ & $P$-values & FDR values \\
\hline pmi-miR398f/g & TGTGTCCTCAGGTCGCCCCCA & 7.05E-7 & $1.07 \mathrm{E}-4$ \\
\hline pmi-miR408 & TGCACTGCCTCTTCCCTGGTTA & $4.58 \mathrm{E}-5$ & $3.76 \mathrm{E}-3$ \\
\hline pmi-miR530 & TATCTGCATTTGCACCTGCACCA & 1.77E-4 & 0.01 \\
\hline pmi-miR530b & TGCATTTGCACCTACACCTTAA & 7.14E-11 & $2.78 \mathrm{E}-8$ \\
\hline pmi-miR535 & TGACAATGAGAGAGAGCATA & $2.73 \mathrm{E}-4$ & 0.02 \\
\hline pmi-miR535a & TाTGACAAAGAGAGAGAGCACGA & 9.67E-4 & 0.04 \\
\hline pmi-miR858 & TTCGTTGTCTGTTCAACCTTA & $1.63 \mathrm{E}-4$ & 0.01 \\
\hline pmi-miR894 & TTTCACGTCGGGTTCATCAA & $1.63 \mathrm{E}-4$ & 0.01 \\
\hline pmi-miR4995 & TAGGCAGTGGCTTGGTTAAGGA & 4.13E-10 & $1.39 \mathrm{E}-7$ \\
\hline pmi-miR5077 & TCGCGTCGGGTTCACCAA & $9.20 \mathrm{E}-4$ & 0.04 \\
\hline pmi-miR5368 & GACAGTCTCAGGTAGACA & 2.61E-10 & $9.11 \mathrm{E}-8$ \\
\hline pmi-miR6173 & AGCCGTAAACGATGGATA & $1.714 \mathrm{E}-4$ & 0.01 \\
\hline pmi-miR6300 & GTCGTTGTAGTATAGTGGA & $2.28 \mathrm{E}-7$ & $4.04 \mathrm{E}-5$ \\
\hline pmi-miR6478 & TCGACCTTAGCTCAGTTGGTA & $6.66 \mathrm{E}-11$ & $2.69 \mathrm{E}-8$ \\
\hline
\end{tabular}

physiological and functional properties to those of terpenoids [30, 31].

\section{Expression profiles of miRNAs and their targets by RT-qPCR} Real-time quantitative polymerase chain reaction (RTqPCR) was performed to experimentally validate the expression of five conserved miRNAs (pmi-miR396a, pmi-
miR398f/g, pmi-miR530, pmi-miR6173, and pmimiR6300) and two novel miRNAs (pmi-nov_12 and pmi-nov_13) and their target transcripts. The expression profiles are shown in Fig. 10. Pmi-miR396a showed upregulation to 2.5 -fold at $9 \mathrm{dpi}$. Its target, peroxidase 57 , was upregulated at $3 \mathrm{dpi}$ and then downregulated at 6 dpi and 9 dpi. Peroxidase is an important enzyme that

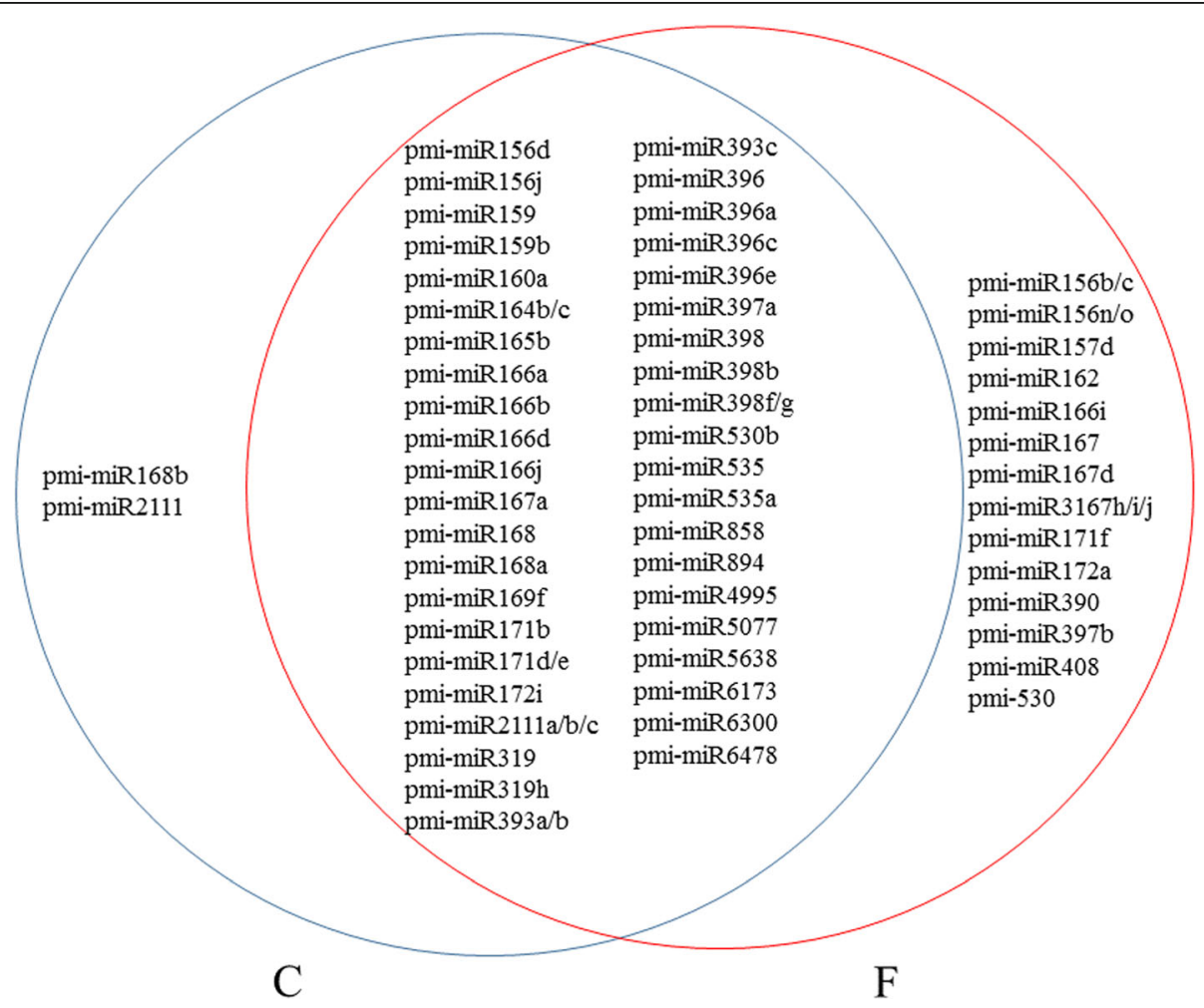

Fig. 6 Common and specific miRNA sequences in the $C$ and $F$ libraries 


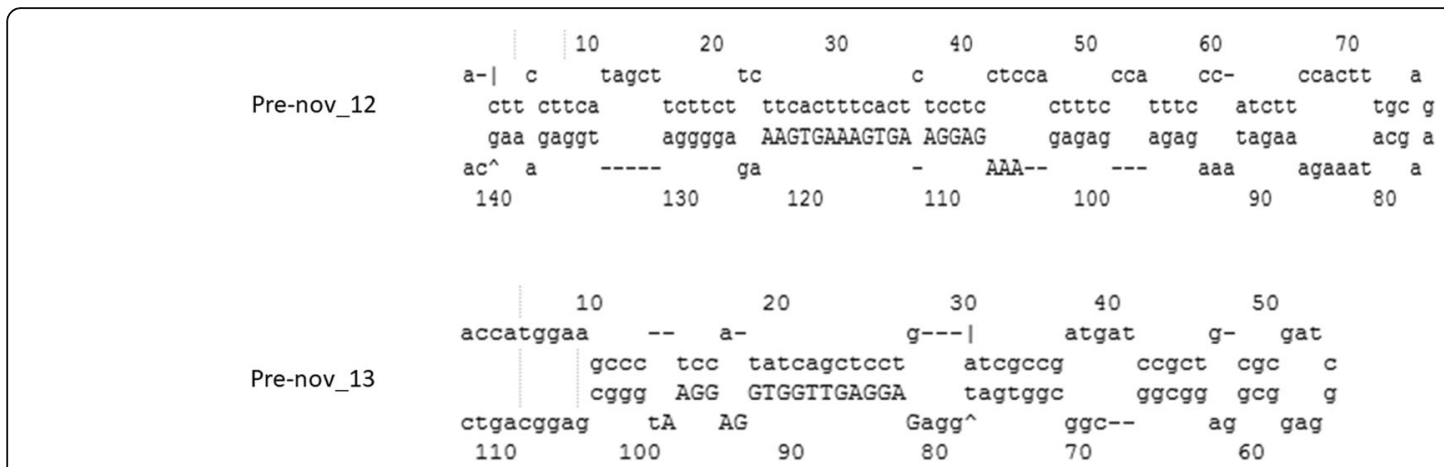

Fig. 7 Stem-loop or pre-miRNA structures for putative novel miRNAs

acts as a scavenger for reactive oxygen species (ROS) [32]. ROS are produced by plants as an early response to stress. Simultaneously, plants require a mechanism to prevent ROS from damaging the cell. Hence, high production of peroxidase during early inoculation (3 dpi) may help neutralize the excessive ROS inside the plant cell. In addition, peroxidase may also be involved in terpenoid biosynthesis. In cucumber inoculated with red spider mites (Tetranychus urticae Koch), increased production of (E, E)- $\alpha$-farnesene resulted [33]. In A. thaliana, miR396 is encoded by two different gene loci, which lead to the biogenesis of two miR396 members, miR396a and miR396b, targeting the GRF transcription factor, which regulates the number of cells in the leaf $[34,35]$. Overexpression of miR396a and miR396b resulted in reduced leaf size [34]. In addition, recent studies have revealed that the application of a target mimic approach in miR396a contributes to plant defence against fungal pathogens. The study suggested that the low activity of miR396a induces the plant defence mechanism through the accumulation of hydrogen peroxidase $\left(\mathrm{H}_{2} \mathrm{O}_{2}\right)$ and callus formation [36]. In rice, a similar approach was used, leading to decreased accumulation of miR396a and increased GRF transcription factor activity. That kind of interaction activates auxin biosynthesis and ARF, thus resulting in greater yield [37]. In addition to peroxidase, another target of pmi-miR396a in P. minor was DXS. Negative correlations between these two were observed at 3 dpi. DXS is involved in the MEP pathway, which produces monoterpene and diterpene as the main products $[9,10]$. The descending pattern of DXS expression corresponds with the increase in pmi-miR396a, especially at $3 \mathrm{dpi}$ and $9 \mathrm{dpi}$. For the target transcript peroxidase 57 , the target was not strongly regulated with high expression of pmi-miR396a at 3 dpi. This kind of regulation was also demonstrated for miR398 in A. thaliana [38]. In A. thaliana, miR398 targeted two types of copper superoxide dismutase (CSD), CSD1 and CSD2. The upregulation of miR398 consistently leads to the downregulation of CSD1, whereas CSD2 did not show any correlation [38].

In $P$. minor, the expression profile of pmi-miR398f/g showed an increasing pattern from $3 \mathrm{dpi}$ to $9 \mathrm{dpi}$, and the maximum was observed at $9 \mathrm{dpi}$. The upregulation of pmi-miR398f/g led to the downregulation of the DXR target transcript. Similar to DXS, DXR is involved in the MEP pathway of terpenoid biosynthesis [10]. The miR398 family is thought to be involved in various plant stress responses by participating in the oxidative burst process $[39,40]$. In $A$. thaliana, the expression of miR398 was downregulated under salinity, oxidative stress, Pseudomonas syringae infection and phosphate deficiency. This downregulation leads to the accumulation of a target transcript, CSD, which acts as a ROS scavenger [39]. In P. minor, pmi-miR398f/g was upregulated upon treatment with $F$. oxysporum. The expression pattern seems to contrast with that of miR398 in $A$. thaliana. However, in Medicago truncatula, miR398a/b,

Table 3 List of novel miRNAs discoveries and their information

\begin{tabular}{|c|c|c|c|c|c|c|c|c|}
\hline ID miRNA ${ }^{a}$ & $\begin{array}{l}\text { miRNA sequence } \\
\left(5^{\prime} \text { to } 3^{\prime}\right)\end{array}$ & Pre-miRNA ID ${ }^{b}$ & $\begin{array}{l}\text { Length of } \\
\text { precursors (nt) }\end{array}$ & ID transcript ${ }^{c}$ & $\% A$ and $\mathrm{U}$ & MFE $(\mathrm{kcal} / \mathrm{mol})^{\mathrm{d}}$ & AMFE $^{\mathrm{e}}$ & $\overline{M F E I^{f}}$ \\
\hline Pmi-nov_12 & AAAGAGGAAGTGAAAGTGAA & Pre-nov_12 & 143 & comp65772_c1_seq1 & 58.04 & -46.40 & 32.45 & 1.30 \\
\hline Pmi-nov_13 & GAGGAGTTGGTGGAGGAA & Pre-nov_13 & 113 & comp63496_c0_seq5 & 35.40 & -49.10 & 43.45 & 1.49 \\
\hline
\end{tabular}




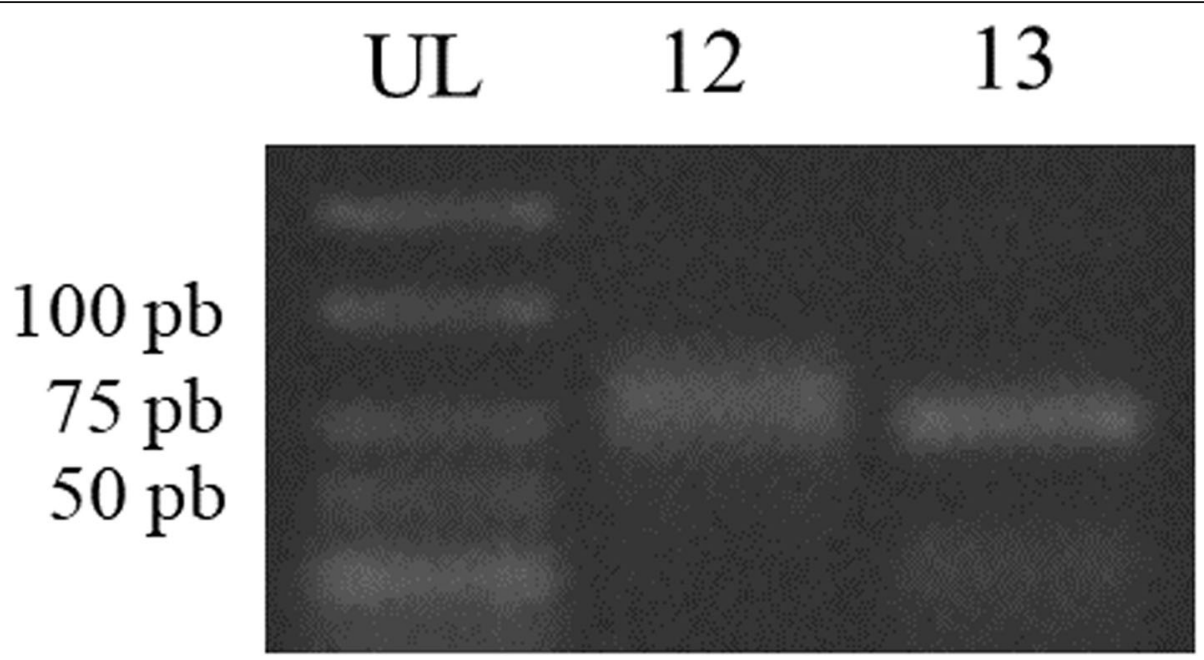

Fig. 8 Experimental validation of miRNA stem-loop structure through RT-PCR

which targets copper-containing proteins involved in copper homeostasis, was upregulated under drought conditions [41].

Pmi-miR6300 showed a decreasing pattern from $3 \mathrm{dpi}$ until $9 \mathrm{dpi}$, resulting in the accumulation of the target transcript, HMGR. The highest accumulation of HMGR was observed at $9 \mathrm{dpi}$, when RT-qPCR showed an upregulation of more than three-fold. HMGR is a vital enzyme involved in sesquiterpene biosynthesis in the MVA pathway [10]. A very limited amount is known about the role of the miR6300 family in plants. In barley, miR6300 was downregulated under drought treatment [42]. In addition, miR6300 has also been discovered in chickpea [43]. However, the target transcripts of miR6300 in both the mentioned studies are still unknown.

Pmi-miR530 targeted MVD, which is involved in the MVA pathway. No significant change was observed in pmi-miR539 expression until $6 \mathrm{dpi}$. On the other hand, the target transcript MVD showed a gradual increase from 3 dpi until 6 dpi. At 9 dpi, pmi-miR530 was drastically upregulated (exceeding 12-fold), while MVD was repressed. The spike in pmi-miR530 expression was supported by a previous study in chickpea. In chickpea inoculated with $F$. oxysporum, there was a 17-fold upregulation of miR530 compared to that in control plants. In addition, miR530 in chickpea targeted zinc knuckle protein, which is involved in regulating plant development [44].

Two target transcripts, FDP and sesquiterpene synthase, which are involved in the MVA pathway, were targeted by pmi-miR6173. RT-qPCR analysis showed a decreasing pattern in pmi-miR6173 expression. The expression patterns for both targets were similar. The expression of target transcripts increased at $3 \mathrm{dpi}$. However, the expression declined at $6 \mathrm{dpi}$, and no significant change was observed from 6 dpi to 9 dpi. A decreasing expression pattern of miR6173 was also observed in the herbs Sedum alfredii and Medicago sativa. In $S$. alfredii, miR6173 was downregulated under cadmium treatment. Target prediction showed that miR6173 in S. alfredii targeted a number of targets, such as calcium-binding EF-hand family protein, ATP synthase subunit $\alpha$, and aspartic protease. However, further work is needed to discover the role of miR6173 in $S$. alfredii [45]. In M. sativa, miR6173 was among the miRNAs downregulated under drought treatment. miR6173 in $M$. sativa targeted splicing factor $3 \mathrm{~A}$ subunit 2, which plays an important role in mRNA splicing [46].

The single gene involved in GLV biosynthesis, $\mathrm{ADH}$, was targeted by the novel miRNA pmi-nov_12. Our results showed that pmi-nov_12 was downregulated from $3 \mathrm{dpi}$ to $6 \mathrm{dpi}$ and then upregulated at $9 \mathrm{dpi}$, where a 1.2-fold increase was observed. The expression pattern of pmi-nov_12 was downregulated and upregulated at a series of time points, showing a dynamic expression pattern. This type of expression was previously reported in Populus tremula. In P. tremula treated with salinity and abscisic acid, miR398 was downregulated in the first 48 $\mathrm{h}$ and then showed high expression at $72 \mathrm{~h}$. In P. minor, although dynamic regulation occurs in pmi-nov_12, a negative correlation can still be observed between pminov_12 and ADH. ADH was reported to be involved in GLV biosynthesis by catalysing the conversion between alcohol and aldehyde via the hydroperoxide lyase pathway [47]. Another novel miRNA in P. minor, pmi-nov_ 13 , was downregulated in response to $F$. oxysporum treatment, and its target transcript (MVK) was upregulated, especially at $6 \mathrm{dpi}$. MVK is involved in the MVA pathway of terpenoid biosynthesis. Notably, pmi-nov_12 and pmi-nov_13 are unique miRNAs in $P$. minor that have never been reported in another plant species 
Table 4 Target prediction for responsive miRNA in $P$. minor against $F$. oxysporum

\begin{tabular}{|c|c|c|c|}
\hline miRNA & Score & ID target & Target annotation \\
\hline \multirow[t]{3}{*}{ pmi-miR156b/c } & 1.0 & comp48942_c0_seq1 & SPL13 \\
\hline & 3.0 & comp66611_c0_seq1 & Ferric reduction oxidase \\
\hline & 3.5 & comp40605_c1_seq1 & F-box kelch repeat \\
\hline \multirow[t]{2}{*}{ pmi-miR156d } & 3.5 & comp52863_c0_seq1 & F-box protein SKIP27 \\
\hline & 3.5 & comp64448_c1_seq76 & Nuclear transcription factor $Y$ subunit $A$ \\
\hline \multirow[t]{3}{*}{ pmi-miR156j } & 2.0 & comp51572_c1_seq2 & SPL4 \\
\hline & 2.0 & comp54862_c0_seq3 & Malate dehydrogenase \\
\hline & 2.5 & comp40605_c1_seq1 & F-box kelch repeat \\
\hline \multirow[t]{3}{*}{ pmi-miR156n/o } & 1.5 & comp52471_c0_seq1 & SPL16 \\
\hline & 3.0 & comp63113_c0_seq2 & bHLH55 \\
\hline & 4.0 & comp52567_c0_seq3 & Probable signal peptidase complex subunit 1 \\
\hline \multirow[t]{2}{*}{ pmi-miR157d } & 3.8 & comp65851_c2_seq1 & Cytochrome P450 81F1 \\
\hline & 4.0 & comp62138_c0_seq4 & Probable carboxylesterase 18 \\
\hline \multirow[t]{2}{*}{ pmi-miR159 } & 1.0 & comp57600_c3_seq1 & Transcription factor GAMYB \\
\hline & 2.0 & comp67380_c0_seq2 & Putative disease resistance protein RGA3 \\
\hline \multirow[t]{2}{*}{ pmi-miR159a } & 2.5 & comp58874_c0_seq4 & WUSHEL \\
\hline & 2.5 & comp65589_c1_seq2 & Uncharacteized protein At2g41620 \\
\hline \multirow[t]{3}{*}{ pmi-miR159b } & 1.5 & comp57600_c3_seq1 & Transcription factor GAMYB \\
\hline & 3.8 & comp59983_c0_seq2 & Mitogen-activated protein kinase kinase kinase 1 \\
\hline & 4.0 & comp61116_c0_seq1 & Transcription factor bHLH140 \\
\hline \multirow[t]{2}{*}{ pmi-miR160a } & 2.5 & comp65542_c1_seq1 & $\begin{array}{l}\text { Probable galacturonosyltransferase-like } \\
5\end{array}$ \\
\hline & 3.5 & comp63097_c0_seq14 & Auxin-responsive protein IAA9 \\
\hline pmi-miR162 & 4.0 & comp61878_c1_seq1 & ABC transporter $C$ family member 3 \\
\hline \multirow[t]{2}{*}{ pmi-miR164b/c } & 3.0 & comp58722_c2_seq1 & Putative disease resistance protein RGA4 \\
\hline & 3.2 & comp64889_c2_seq23 & E3 ubiquitin-protein ligase RNF8-A \\
\hline \multirow[t]{3}{*}{ pmi-miR165b } & 3.5 & comp12615_c0_seq1 & Putative F-box protein At3g10240 \\
\hline & 3.8 & comp31134_c0_seq2 & F-box protein At3g44326 \\
\hline & 3.8 & comp62577_c0_seq2 & Probable galacturonosyltransferase 12 \\
\hline \multirow[t]{2}{*}{ pmi-miR166a } & 1.5 & comp62172_c1_seq10 & Homeobox-leucine zipper protein HOX32 \\
\hline & 3.5 & comp67610_c2_seq1 & Probable WRKY transcription factor19 \\
\hline pmi-miR166b & 4.0 & comp66580_c3_seq9 & Serine/threonine-protein kinase $\mathrm{TIO}$ \\
\hline \multirow[t]{2}{*}{ pmi-miR166d } & 2.8 & comp62172_c1_seq1 & Homeobox-leucine zipper protein HOX32 \\
\hline & 4.0 & comp23266_c0_seq1 & Probable disease resistance protein At5g63020 \\
\hline \multirow[t]{3}{*}{ pmi-miR166i } & 2.0 & comp67539_c0_seq10 & Probable LRR receptor-like protein kinase At1g51890 \\
\hline & 2.5 & comp58141_c0_seq2 & NADH dehydrogenase (ubiquinone) complexl \\
\hline & 3.5 & comp67658_c0_seq4 & Programmed cell death protein 4 \\
\hline \multirow[t]{2}{*}{ pmi-miR166j } & 1.8 & comp62172_c1_seq12 & Homeobox-leucine zipper protein ATHB-8 \\
\hline & 4.0 & comp60418_c0_seq2 & Protein caperon dnaJ15 \\
\hline \multirow[t]{2}{*}{ pmi-miR167 } & 2.5 & comp65895_c0_seq14 & Probable glycosyltransferase At5g03795 \\
\hline & 3.5 & comp63430_c0_seq5 & Tubulin a-5 chain \\
\hline \multirow[t]{2}{*}{ pmi-miR167a } & 2.0 & comp67548_c0_seq10 & 265 protease regulatory subunit $6 \mathrm{~B}$ homolog \\
\hline & 4.0 & comp22416_c0_seq1 & Putative ribonuclease H Protein At1g65750 \\
\hline pmi-miR167d & 4.0 & comp58506_c2_seq5 & Pre-mRNA-splicing factor SF2 \\
\hline
\end{tabular}


Table 4 Target prediction for responsive miRNA in P. minor against F. oxysporum (Continued)

\begin{tabular}{|c|c|c|c|}
\hline miRNA & Score & ID target & Target annotation \\
\hline & 4.0 & comp67417_c0_seq18 & Binding protein DNA BIN4 \\
\hline \multirow[t]{3}{*}{ pmi-miR167h/i/j } & 2.5 & comp65003_c0_seq2 & AR6 HPI \\
\hline & 3.2 & comp52198_c0_seq1 & E3 ubiquitin protein ligase DRIP1 \\
\hline & 3.8 & comp60694_c0_seq1 & Probable mediator of RNA polymerase II transcription subunit 26b \\
\hline \multirow[t]{2}{*}{ pmi-miR168 } & 3.5 & comp2671_c0_seq1 & Uncharacterized mitochondrial protein AtMg00820 \\
\hline & 3.5 & comp65213_c0_seq4 & Putative F-box protein At3g47150 \\
\hline pmi-miR168a & 3.5 & comp67057_c0_seq6 & Guard cell S-type anion channel SLAC1 \\
\hline pmi-miR168b & 3.8 & comp33471_c0_seq1 & U-box domain-containing protein 36 \\
\hline \multirow[t]{2}{*}{ pmi-miR169f } & 3.0 & comp65145_c0_seq1 & Serine/threonine-protein kinase ppk 15 \\
\hline & 3.2 & comp60891_c3_seq1 & Nuclear transcription factor $Y$ subunit $A$ \\
\hline \multirow[t]{2}{*}{ pmi-miR171b } & 1.5 & comp59653_c0_seq3 & SCL6 \\
\hline & 2.5 & comp68032_c2_seq2 & Putative disease resistance protein RGA4 \\
\hline \multirow[t]{3}{*}{ pmi-miR171d/e } & 1.8 & comp59653_c0_seq3 & SCL6 \\
\hline & 2.2 & comp48883_c1_seq1 & Uncharacterized mitochondrial protein AtMg01060 \\
\hline & 3.8 & comp58120_c1_seq1 & Transmembrane protein 214-A \\
\hline \multirow[t]{2}{*}{ pmi-miR171f } & 2.0 & comp59653_c0_seq3 & SCL6 \\
\hline & 2.5 & comp68032_c2_seq3 & Putative disease resistance protein RGA4 \\
\hline pmi-miR172a & 1.5 & comp52285_c0_seq1 & AP2 \\
\hline \multirow[t]{3}{*}{ pmi-miR172i } & 3.5 & comp57377_c0_seq5 & Transcription factor NAC29 \\
\hline & 3.8 & comp63311_c0_seq3 & Transcription factor CAULIFLOWER \\
\hline & 4.0 & comp33332_c0_seq1 & E3 ubiquitin-protein ligase makorin \\
\hline \multirow[t]{2}{*}{ pmi-miR2111 } & 3.0 & comp29780_c0_seq1 & Probable WRKY transcription factor 7 \\
\hline & 3.5 & comp41575_c0_seq2 & Putative ribonuclease $\mathrm{H}$ protein At1g65750 \\
\hline \multirow[t]{3}{*}{ pmi-miR2111a/b/c } & 2.5 & comp41652_c0_seq2 & Transcription factor WRKY55 \\
\hline & 3.2 & comp59219_c1_seq1 & Actin-related protein 6 \\
\hline & 3.5 & comp63701_c0_seq2 & Transcription factor bHLH51 \\
\hline pmi-miR319 & 3.8 & comp61400_c1_seq1 & Probable sulfate transporter 3.5 \\
\hline pmi-miR319h & 4.0 & comp41320_c0_seq1 & Small heat shock protein \\
\hline \multirow[t]{2}{*}{ pmi-miR390 } & 3.8 & comp68004_c1_seq12 & Putative disease resistance protein RGA4 \\
\hline & 3.8 & comp68227_c1_seq27 & AGO5 \\
\hline \multirow[t]{2}{*}{ pmi-miR393c } & 3.2 & comp60765_c4_seq1 & Proteasome subunit a type-1-A \\
\hline & 3.8 & comp64012_c0_seq1 & Pectinesterase31 \\
\hline pmi-miR396 & 2.5 & comp12732_c0_seq1 & Putative ribonuclease H protein At1g65750 \\
\hline \multirow[t]{2}{*}{ pmi-miR396a } & 2.5 & comp60490_c0_seq1 & Peroxidase57 \\
\hline & 4.0 & comp47449_c0_seq1 & Probable DXS \\
\hline pmi-miR396c & 2.5 & comp47994_c0_seq1 & Hypersensitive-induced response protein 1 \\
\hline \multirow[t]{2}{*}{ pmi-miR396e } & 3.2 & comp58032_c1_seq1 & Cytochrome b \\
\hline & 3.5 & comp60164_c0_seq2 & Putative F-box protein At3g23950 \\
\hline pmi-miR397a & 0.8 & comp67947_c0_seq1 & Laccase-4 \\
\hline pmi-miR397b & 1.5 & comp67947_c0_seq1 & Laccase-4 \\
\hline pmi-miR398 & 3.5 & comp65120_c0_seq4 & L-ascorbate oxidase \\
\hline \multirow[t]{2}{*}{ pmi-miR398b } & 3.8 & comp63418_c1_seq1 & Homeobox-leucine zipper protein ANTHOCYANINLESS2 \\
\hline & 4.0 & comp34288_c0_seq1 & Cellulose synthase-like protein D3 \\
\hline pmi-miR398f/g & 4.0 & comp67631_c2_seq15 & DXR \\
\hline
\end{tabular}


Table 4 Target prediction for responsive miRNA in P. minor against F. oxysporum (Continued)

\begin{tabular}{|c|c|c|c|}
\hline miRNA & Score & ID target & Target annotation \\
\hline pmi-miR408 & 1.5 & comp50583_c0_seq1 & Basic blue protein \\
\hline \multirow[t]{2}{*}{ pmi-miR530 } & 4.0 & comp31767_c1_seq1 & MVD \\
\hline & 4.0 & comp56913_c1_seq1 & Probable sulphate transporter \\
\hline pmi-miR530b & 3.0 & comp66662_c8_seq34 & $50 S$ ribosomal protein $L 2$ \\
\hline pmi-miR535 & 2.5 & comp53737_c0_seq1 & Ferredoxin-thioredoxin reduktase catalytic chain \\
\hline \multirow[t]{2}{*}{ pmi-miR535a } & 3.2 & comp51270_c0_seq1 & Transcription factor TCP8 \\
\hline & 3.5 & comp55340_c0_seq1 & Probable cellulose synthase A catalytic subunit 5 \\
\hline \multirow[t]{2}{*}{ pmi-miR858 } & 3.8 & comp47431_c1_seq1 & E3 ubiquitin-protein ligase ATL31 \\
\hline & 4.0 & comp55416_c0_seq1 & Callos synthase 10 \\
\hline \multirow[t]{3}{*}{ pmi-miR894 } & 2.0 & comp66746_c0_seq1 & U-box domain-containing protein 13 \\
\hline & 2.5 & comp67024_c1_seq10 & Heat shock $70 \mathrm{kDa}$ protein 16 \\
\hline & 4.0 & comp18439_c0_seq1 & Polygalacturonase At1g48100 \\
\hline pmi-miR4995 & 3.0 & comp60227_c1_seq1 & Probable WRKY transcription factor 39 \\
\hline \multirow[t]{2}{*}{ pmi-miR5077 } & 3.2 & comp30119_c0_seq1 & Ethylene-responsive transcription factor ERO HPI25 \\
\hline & 3.5 & comp63610_c1_seq2 & Cellulose synthase A catalytic subunit 7 \\
\hline pmi-miR5368 & 3.8 & comp12493_c0_seq1 & Sucrose synthase 5 \\
\hline \multirow[t]{2}{*}{ pmi-miR6173 } & 3.0 & comp46206_c0_seq1 & Sesquiterpene synthase \\
\hline & 4.0 & comp62238_c1_seq1 & Farnesyl diphosphate synthase 1 \\
\hline \multirow[t]{2}{*}{ pmi-miR6300 } & 3.2 & comp55945_c0_seq1 & HMGR \\
\hline & 3.5 & comp59913_c0_seq1 & Proteasome subunit beta type-2-A \\
\hline pmi-miR6478 & 3.8 & comp62172_c1_seq14 & Homeobox-leucine zipper protein HOX33 \\
\hline pmi-nov_12 & 2.5 & comp58932_c3_seq6 & Alcohol dehydrogenase \\
\hline \multirow[t]{2}{*}{ pmi-nov_13 } & 2.5 & comp65932_c1_seq10 & Mitogen-activated protein kinase 16 \\
\hline & 3.8 & comp56286_c0_seq1 & Mevalonate kinase \\
\hline
\end{tabular}

before. However, for pmi-miR6173, pmi-nov_12 and pmi-nov_13, the expression of the target genes was comparable to miRNA expression even with decreasing metabolite content at the end of the treatment $(9 \mathrm{dpi})$. Hence, we suggest that these miRNAs may inhibit mRNA translation, which is another mode of miRNA action $[48,49]$.

miRNAs as post-transcriptional regulator in $P$. minor In plants, miRNAs play roles in various biological processes, such as plant development, signal transduction, stress response, and secondary metabolite regulation [50]. In this study, miRNAs were discovered that regulate both the MVA and MEP pathways in terpenoid biosynthesis in P. minor (Fig. 11). Four miRNAs, pmimiR6300, pmi-nov_12, pmi-miR530, and pmi-miR6173, were found to be involved in the MVA pathway, whereas two miRNAs, pmi-miR396a and pmi-miR398f/g, were found to be involved in the MEP pathway. Most of the miRNAs regulate upstream genes in the terpenoid biosynthesis pathway.

In the MVA pathway, among the four responsive miRNAs, three (pmi-miR6300, pmi-6173 and pmi-nov_13) were downregulated, whereas the fourth miRNA (pmimiR530) showed a sharp increase at $9 \mathrm{dpi}$. The PmimiR6300-regulated gene encoded HMGR, a rate-limiting enzyme in the MVA pathway and the first rate-limiting enzyme recognized in the terpenoid biosynthesis pathway [51]. In A. thaliana, the HMGR gene has undergone a duplication process that led to HMG1 and HMG2. HMG1 is expressed throughout the plant, whereas the expression of HMG2 was observed in only the meristem and floral parts of $A$. thaliana plants [51]. In addition, the expression of HMGR is affected by internal factors such as plant development and phytohormone [52]. HMGR is also affected by external stimuli, such as light, wounding, elicitor treatment and pathogen invasion $[52,53]$. The mutant $h m g 1$ in $A$. thaliana exhibited infertility, premature senescence, and dwarfness [54]. In the MVA pathway, HMGR catalyses the rate-limiting steps that directly affect downstream products. In $A$. thaliana, the hmg1 mutant showed a $65 \%$ reduction in triterpene compound accumulation compared to the wild type [55]. Moreover, elicitor treatments such as fungal inoculation and wounding also increased the expression level of HMGR and subsequently led to higher accumulation of sesquiterpene compounds 


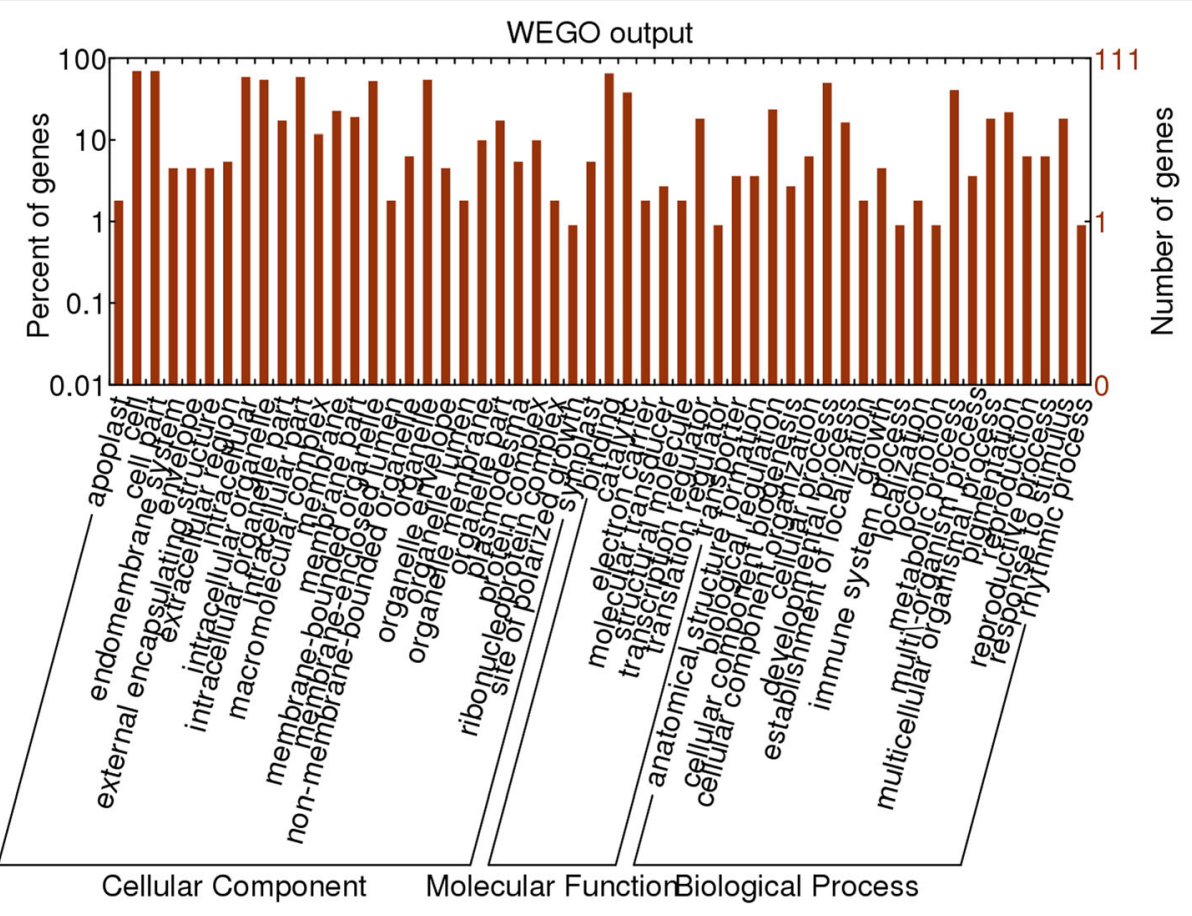

Fig. 9 Functional classification of target transcripts by WEGO software

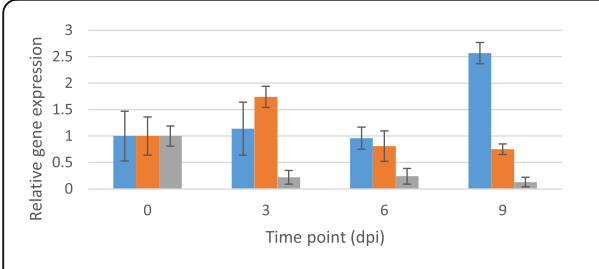

- pmi-miR396a |- Peroxidase57 $=$ DXS

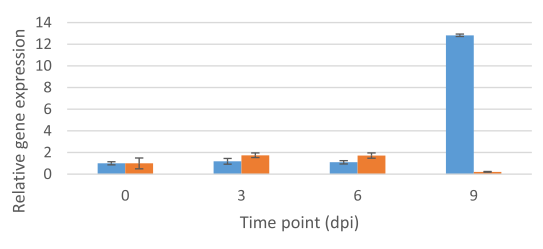

apmi-miR530 $=\mathrm{MVD}$

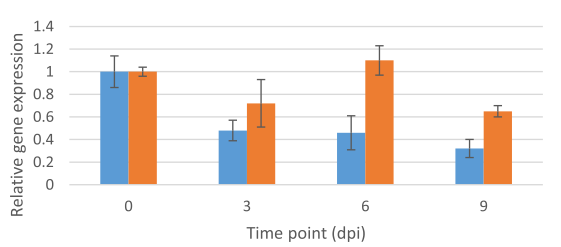

- pmi-nov_13 $=$ MVK

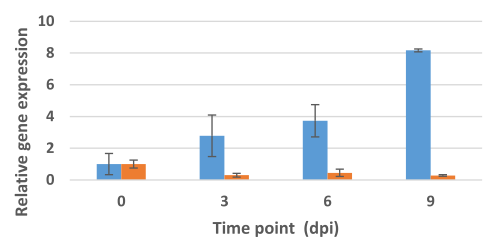

- pmi-miR398f/g n DXR

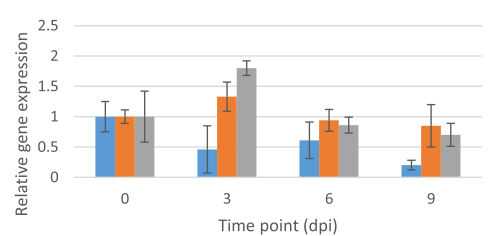

mpmi-miR6173 $\quad$ - FDS1 $\quad$ - Sesquiterpene synthase

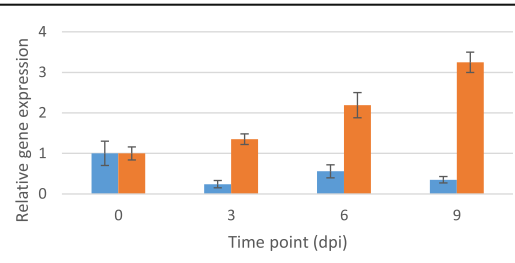

- pmi-miR6300 = HMGR

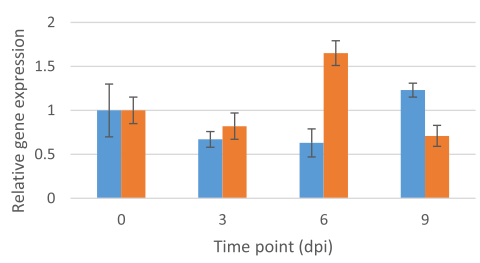

apmi-nov_12 $=\mathrm{ADH}$

Fig. 10 Relative expression of miRNAs with respect to their target transcripts 


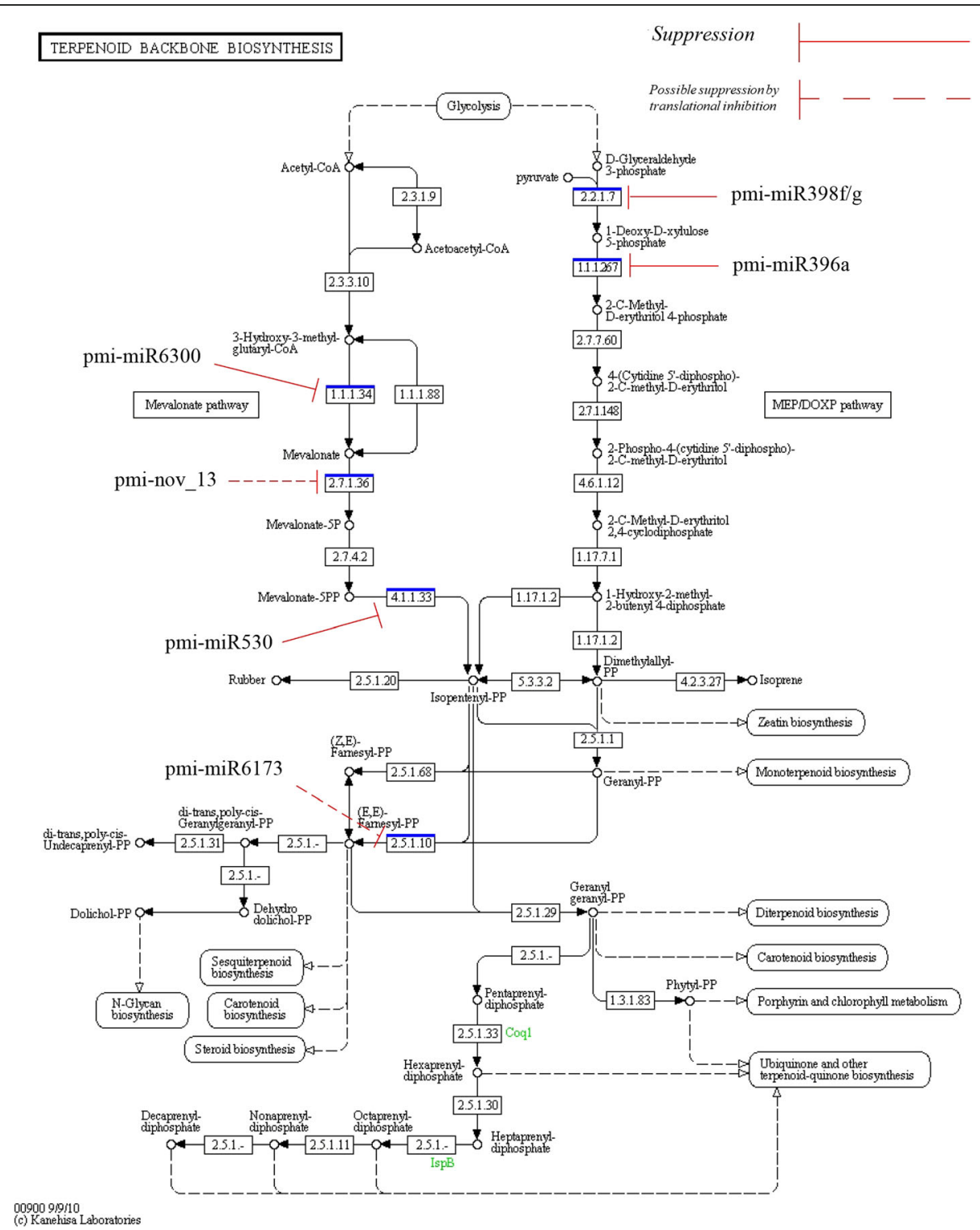

Fig. 11 Involvement of miRNAs in the terpenoid pathway in P. minor EC 2.2.1.7: 1-deoxy-D-xylulose-5-phosphate synthase; EC 1.1.1.267: 1-deoxyD-xylulose-5-phosphate reductoisomerase; EC 1.1.1.34: hydroxymethylglutaryl-CoA reductase; EC 2.7.1.36: mevalonate kinase; EC 4.1.1.33: diphosphomevalonate decarboxylase; EC 2.5.1.10: farnesyl diphosphate synthase. The terpenoid biosynthesis backbone pathway was constructed using KEGG software. Suppression symbol (continuous line) indicate the miRNAs had displayed negative relationship against their own target, while dashed suppression symbol indicate the hypothetical effect of miRNAs to inhibit the target via translational inhibition

$[53,56]$. In P. minor, terpenoids are produced in small quantities under normal conditions. RT-qPCR results showed that pmi-miR6300 was upregulated at $0 \mathrm{dpi}$, which led to the suppression of its target, HMGR. Indeed, after treatment, pmi-miR6300 was downregulated and led to the high accumulation of HMGR. In $X$. strumarium, HMGR was targeted by miR1134 and miR5021 [50]. However, the study of both miRNA and HMGR in X. strumarium is still at the prediction level and requires further experimental validation. In the MVA pathway, the HMGR enzyme catalyses the conversion of HMG-CoA to mevalonate, which is later converted into mevalonate-5-phosphate through the enzyme MVK. Until now, no clear interaction between miRNA and MVK has been reported in plants, while in mice, miR122 targets MVK, which is involved in cholesterol biosynthesis $[1,57]$. In this study, we investigated the interaction and reported that pmi-nov_13 targets MVK in $P$. minor. The second miRNA, pmi-miR530, was found to regulate the MVD enzyme, which catalyses mevalonate diphosphate into IPP. Pmi-miR530 was increased drastically at $9 \mathrm{dpi}$, which led to the downregulation of its target. As for pmi-nov_13 and MVK, no miRNA in plants has previously been reported to 
target MVD. In mice, miR124 is involved in hypocholesterolaemia by targeting MVD [58]. In plants, information about the roles of MVK and MVD is quite limited compared to yeast [59]. However, the latest study of MVK in Gingko biloba discovered that overexpression of MVK led to the accumulation of the terpene trilactone [60]. In P. minor, the accumulation of MVK at 6 dpi may contribute to sesquiterpene biosynthesis, although the rate-limiting enzyme had its highest expression at $9 \mathrm{dpi}$.

In the MEP pathway, two miRNAs (pmi-miR396a and miR398f/g) were discovered to be involved by targeting DXS and DXR. DXS is involved in the upstream reaction in the MEP pathway by catalysing the condensation process between pyruvate and D-glyceraldehyde 3phosphate to produce DXP [61]. The discovery of DXS was first reported in $E$. coli and then in A. thaliana via mutation of the cla gene $[61,62]$. Transgenic A. thaliana overexpressing the DXS gene resulted in an abundance of terpenoids compared to wild plants, and DXS was categorized as a rate-limiting enzyme in the MEP pathway [61]. Further study of DXS in A. thaliana showed that DXS exists as the paralogues DSX1 and DXS2. Of these two paralogues, only DXS1 was found to be involved in terpenoid biosynthesis [63].

In $A$. thaliana, the majority of the DXS genes were expressed in photosynthetic organs, such as leaves, and floral parts, while homologues to DXS2 in tomato were expressed in roots and trichomes [64]. In the second step of the MEP pathway, the DXR enzyme converts DXP to MEP via rearrangement of the molecular structure followed by a reduction process by NADPH. This step is reversible. Unlike DXS, the role of DXR as a ratelimiting enzyme is still unclear, and its function may depend on plant species, organ and development stage [65]. However, similar to DXS, DXR genes are distributed in different plant parts and stimulated by light response [65]. The mutant $d x r$ exhibited an albino phenotype, defects in gibberellin and abscisic acid biosynthesis and improper formation of trichomes and stomatal closure [66].

Interestingly, in P. minor, the majority of miRNAs involved in the MVA pathway were downregulated except for pmi-miR530, which was upregulated at $9 \mathrm{dpi}$. The decreasing pattern exhibited by pmi-miR6300, pmi-nov_ 13 and pmi-miR6173 resulted in the accumulation of their target transcripts, which most likely regulate sesquiterpene production. These findings were supported by the metabolite profile in Fig. 2, which shows prominent production of $\alpha$-cedrene, valencene, and $\beta$ bisabolene after treatment with $F$. oxysporum. Meanwhile, the increasing pattern shown by pmi-miR396a and pmi-miR398f/g resulted in the suppression of DXS and DXR in the MEP pathway. The differences in miRNA expression involved in regulating MVA and
MEP are probably due to the effect of $F$. oxysporum, which stimulates terpenoid production in the MVA rather than the MEP pathway. Downstream products in the MVA pathway (sesquiterpene), which play crucial roles in plant defence mechanisms, may influence the selection of the terpenoid pathway in the plant system. In addition, the interaction of two regulators, miRNAs and transcription factors, can also affect terpenoid biosynthesis. In $A$. thaliana and $P$. cablin, expression of miR156 reduced the production of sesquiterpene by suppression of the target, SPL [67]. In P. minor, pmi-miR156b/c was predicted to target the SPL gene. However, there is no evidence that this interaction would affect sesquiterpene production, because a previous report showed that the interaction between miR156 and SPL is conserved only in regulating floral development $[11,68]$.

miRNA cleavage site determination by degradome analysis Degradome sequencing was carried out to determine the miRNA cleavage site. The sequences were deposited under accession number SRX3921398. The statistical data are shown in Table 5. There were 17,532,759 degradome sequencing reads. Filtering and removal of adaptors revealed 15,493,710 clean reads. Mapping to the $P$. minor transcriptome resulted in $3,079,840$ sequence tags. However, to increase the hit search, another $P$. minor degradome library, with the accession number SRX3921610, was used together with this degradome library. The results showed that out of the 7 studied miRNAs involved in the terpenoid biosynthesis pathway, cleavage sites for pmimiR396a and pmi-miR6300 and their targets, peroxidase57 and HMGR, were detected between positions 10 and 11 of the miRNA sequences (Fig. 12), and this result was also supported by previous findings [28, 29].

\section{Spearman correlation analysis}

Overall, correlation analysis revealed that pmi-miR530 and pmi-miR398f/g had positive correlations with metabolite content, whereas the remaining miRNAs (pmi-396a, pmimiR6300, pmi-miR6173 and pmi-nov_13) showed negative correlations (Fig. 13a). Pmi-nov_12 was excluded from this analysis because it was not involved in the terpenoid biosynthetic pathway. For the target genes, the majority of them displayed positive correlations with metabolite content, except for HMGR (Fig. 13b). The low abundance of $\alpha$ cedrene, valencene and $\beta$-bisabolene at 9 dpi may have

Table 5 Statistic for degradome sequencing

\begin{tabular}{lll}
\hline & Total reads & Percentages (\%) \\
\hline Raw reads & $17,532,759$ & \\
Clean reads & $15,493,710$ & 100.00 \\
Sequence tag & $3,074,840$ & 19.85 \\
Discard reads & $12,418,870$ & 80.15 \\
\hline
\end{tabular}




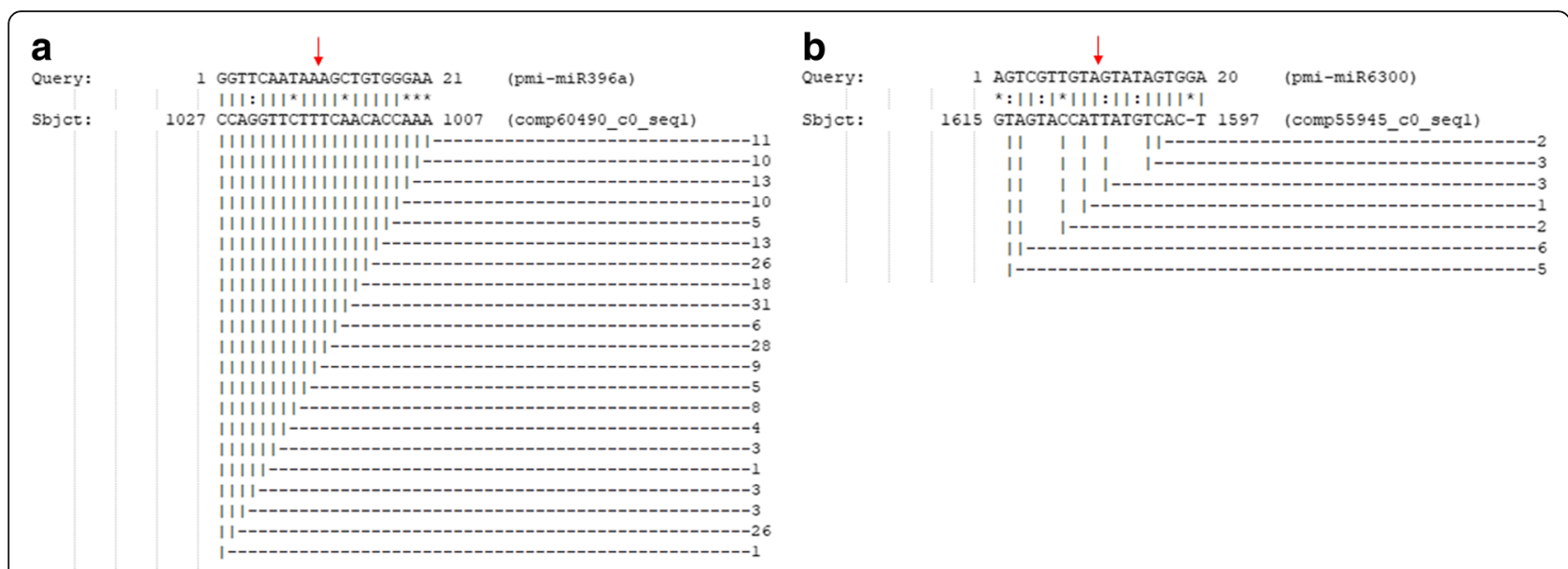

Fig. 12 Detection of miRNA cleavage sites. The number of sequence tags is shown on the right side. The arrows indicate the positions where the miRNAs cleave the targets. a shows the cleavage site for pmi-miR396a, while $\mathbf{b}$ shows the cleavage site for pmi-miR6300

contributed to this negative correlation because HMGR expression continued to increase during the fungus treatment. The Spearman coefficients are included in Additional file 3.

\section{Conclusion}

In this study, a total of 58 conserved and two novel miRNAs responsive to $F$. oxysporum treatment in $P$. minor were identified. Five of the 58 conserved miRNAs and a novel miRNA were found to be directly involved with the MVA and MEP pathways. However, some of the miRNAs, such as pmi_miR6173, pmi-nov_12 and pmi-nov_13, might need further confirmation at the protein level against their own targets. This work provides the framework for further exploration of miRNA-mediated regulatory mechanisms in terpenoid biosynthesis in $P$. minor. Moreover, our study also found some miRNAs targeting mRNAs encoding transcription factors, which suggested the role of miRNAs in regulating various biological processes, including plant growth and development. The above seven studied miRNAs could be utilized to regulate secondary metabolite biosynthesis by manipulating the MVA and MEP pathways through the RNAi mechanism.

\section{Methods}

\section{Plant and fungus cultures}

P. minor plants were cultured for approximately 6 weeks on Murashige and Skoog media in a controlled chamber room at Kompleks Rumah Tumbuhan, Universiti
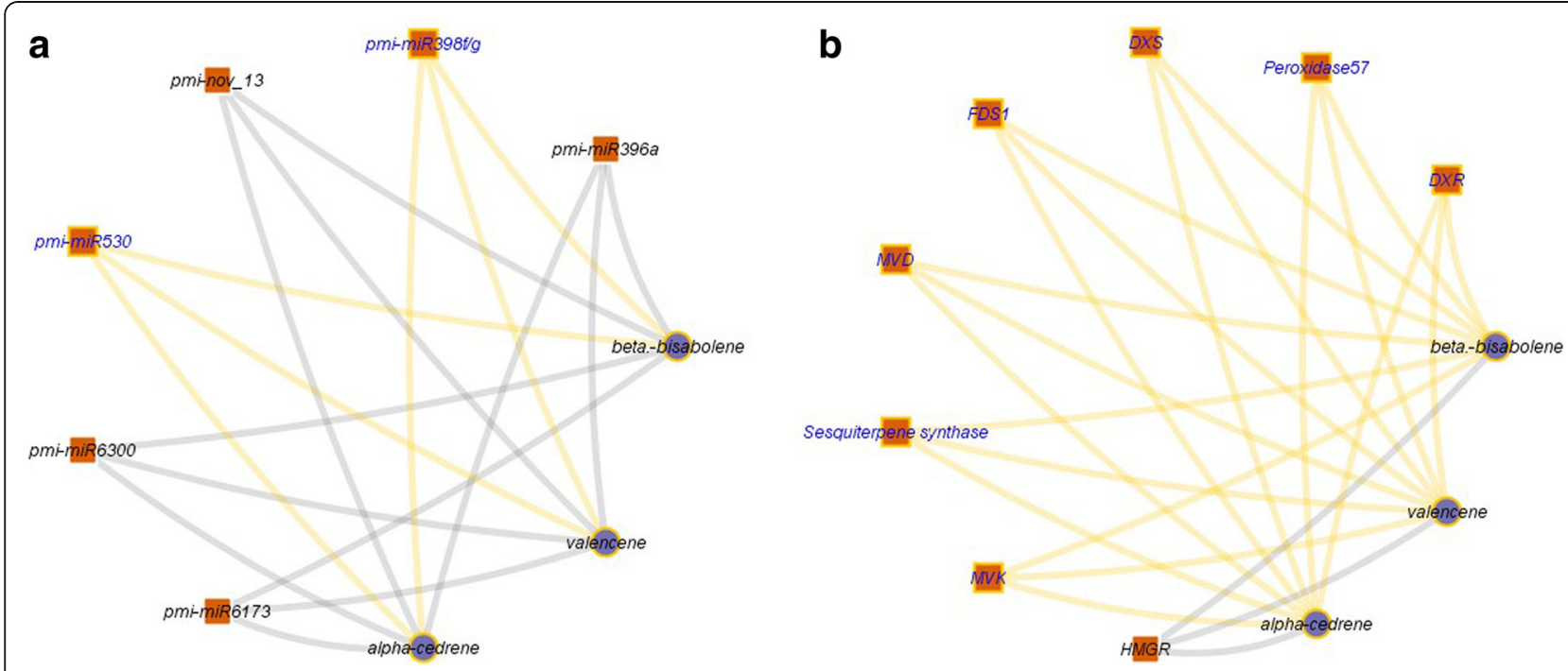

Fig. 13 Spearman correlation analysis. Correlation analysis between miRNA-metabolites (a) and mRNA-metabolites (b). In A, miRNAs are represented by squares, whereas metabolites are represented by circles. In B, mRNAs are represented by squares, whereas metabolites are represented by circles. In both figures, yellow lines indicate positive correlations, and grey lines indicate negative correlations 
Kebangsaan Malaysia ( $\left.3^{\circ} 16^{\prime} 14.63 " \mathrm{~N}, 101^{\circ} 41^{\prime} 11.32^{\prime \prime} \mathrm{E}\right)$ [69]. The F. oxysporum fungal culture was obtained from a microbe collection room at the School of Biosciences and Biotechnology, Universiti Kebangsaan Malaysia. Small pieces of the fungal culture were cut and transferred to PDA plates. Two-week-old $F$. oxysporum culture was used for the inoculation.

\section{F. oxysporum inoculation and terpenoid profiling analysis} Prior to treatment, $P$. minor plants were transferred from solid to liquid MS media. The plant culture was maintained in a controlled chamber overnight. F. oxysporum spores were collected by rinsing the PDA with sterile distilled water. Approximately $0.05 \%$ of Tween 20 was added to the spore solution. The concentration of $F$. oxysporum was adjusted to $5 \times 10^{7} / \mathrm{mL}$ before being added to the plant culture. The treated plants were kept in a controlled room with $16 \mathrm{~h}$ light and $8 \mathrm{~h}$ dark.

Metabolite profiling in $P$. minor was carried out at a series of time points, beginning with 3 days postinoculation (dpi), $6 \mathrm{dpi}$, and $9 \mathrm{dpi}$, and using SPMEGCMS. In addition, $P$. minor plants treated with sterile distilled water were used as a control ( 0 dpi). Every time point had three biological replicates. The SPME method was adapted for the collection of volatiles. P. minor leaves were harvested (approximately $1 \mathrm{~g}$ ) in liquid nitrogen and ground into small pieces. The ground tissues were then promptly transferred into labelled SPME vials to avoid evaporation of volatile compounds. The vials were closed with screw caps and then heated at $65{ }^{\circ} \mathrm{C}$ for $15 \mathrm{~min}$ in a water bath. Volatile compounds were collected by introducing an SPME needle via a septum cap. SPME fibre $(100 \mu \mathrm{M}$ polydimethylsiloxane, PDMS), which was located inside the SPME needle, was injected into the vials to absorb the volatiles. Alternatively, polyacrylate fiber also could be used for volatiles detection [70]. The equilibrium time for SPME fibre in the collection vial was set for $30 \mathrm{~min}$ at $55^{\circ} \mathrm{C}-60^{\circ} \mathrm{C}$. GC-MS analysis was performed on an Agilent 7890A gas chromatograph (GC) directly coupled to the mass spectrophotometer (MS) of an Agilent 5975C inert MSD with a triple axis detector. The column used was a non-polar column, HP-5MS (30 m length $\times 0.25 \mathrm{~mm}$ ) diameter and film thickness $0.25 \mu \mathrm{M}$. Helium was used as the carrier gas, with a flow rate of 1.3 $\mathrm{mL} / \mathrm{min}$. A splitless injection was set at $50^{\circ} \mathrm{C}$ hold for 3 min, increased to $100^{\circ} \mathrm{C}$ at a rate of $20^{\circ} \mathrm{C} /$ minutes, and held at $250^{\circ} \mathrm{C}$ for $3 \mathrm{~min}$. The peaks were identified by searching the NIST/EPA/NIH mass spectral library (version 11 , and the results were combined in a GC-MS chromatogram.

\section{Statistical analysis of metabolite profiling}

The metabolite profiling was analysed using MetaboAnalyst software (http://www.metaboanalyst.ca) version 3.0 [71].
The data were normalized according to the sum from each sample. One-way ANOVA was carried out followed by Fisher's LSD test. The $P$-value was set at $P<0.05$.

\section{Small RNA library construction}

To generate high-quality small RNA libraries, the crucial step is to obtain high-quality total RNA. Low-quality starting material can reduce the quantity of small RNA [72]. Total RNA was extracted, and the quality (purity and concentration) was measured using a Nanodrop spectrophotometer (ND-1000) and Qubit, respectively. The integrity of the extracted RNA was determined by Bioanalyzer analysis (Agilent 2100) using an RNA 6000 chip. RNA samples with RNA integrity number (RIN) values over 7.0 were selected for small RNA library construction. All quality control steps were enclosed in Additional file 2. Mock-inoculated (C) and Fusariumtreated (F) small RNA libraries were constructed with two biological replicates, $\mathrm{C} 1$ and $\mathrm{C} 2$, and F1 and F2, respectively. For the F library, the time point at which $P$. minor emitted volatiles at the maximum level was selected for small RNA library construction. Mockinoculated $P$. minor were prepared by adding sterile distilled water to the plant and used as a control. RNA from both the $\mathrm{C}$ and $\mathrm{F}$ samples was extracted using PureLink ${ }^{\bullet}$ Plant RNA Reagent (Thermo Fisher Scientific, USA) using the recommended protocol. Briefly, approximately $0.1 \mathrm{~g}$ of leaf sample from $P$. minor was ground and transferred to $1.5 \mathrm{~mL}$. A volume of $0.5 \mathrm{~mL}$ of PureLink $^{\odot}$ Plant RNA Reagent was added and vortexed. The samples were incubated at room temperature for $5 \mathrm{~min}$. Next, the samples were centrifuged at $12,000 \times \mathrm{g}$ for 2 min at $4{ }^{\circ} \mathrm{C}$. Approximately $450 \mu \mathrm{L}$ of supernatant was transferred into new tubes, and $0.1 \mathrm{~mL}$ of $5 \mathrm{M} \mathrm{NaCl}$ and $0.3 \mathrm{~mL}$ of chloroform were added and mixed to the supernatant. The mixtures were centrifuged at $12,000 \times \mathrm{g}$ for $2 \mathrm{~min}$ at $4{ }^{\circ} \mathrm{C}$, and approximately $350 \mu \mathrm{L}$ of supernatant was transferred to new tubes, mixed, and incubated for $10 \mathrm{~min}$ at room temperature. The tubes were then centrifuged at $12,000 \times \mathrm{g}$ for $2 \mathrm{~min}$ at $4{ }^{\circ} \mathrm{C}$, and a small pellet was observed at the bottom of each tube. The supernatant was discarded, and $75 \%$ ethanol was added to wash the pellet. Then, the tubes were centrifuged again at $12,000 \times \mathrm{g}$ for $1 \mathrm{~min}$ at $4{ }^{\circ} \mathrm{C}$. The ethanol was discarded, and the pellet was diluted with $20 \mu \mathrm{L}$ of nuclease-free water. Then, DNase treatment was carried out for each RNA sample. The concentration of RNA was determined using a Nanodrop spectrophotometer (Thermo Scientific, USA). The integrity of the RNA was assessed by running a $1 \%$ agarose gel and Bioanalyzer analysis (Agilent 2100 Bioanalyzer, USA). A small RNA library was constructed using the NEBNext Small RNA Library preparation kit as reported by a previous study [22]. Then, 
the libraries were sent to Universiti Malaya, Malaysia for sequencing using an Illumina HiSeq $2500^{\mathrm{m}}$ in Rapid Run mode.

\section{Bioinformatic analysis}

Raw data were analysed using CLC Genomics Workbench version 8 [69]. The adaptors were removed, and the low-quality reads were filtered. Only clean reads with lengths between $18 \mathrm{nt}$ and $30 \mathrm{nt}$ were selected for further analysis. The annotation process was carried out by mapping the clean reads to the miRNA and non-coding-RNA databases miRBase version 21 and Rfam version 12 [23, 73]. A maximum of two mismatches were allowed [74]. Then, each library was 11normalized to transcripts per million (TPM); normalized expression $=($ actual miRNA count $/$ total count of clean reads) $\times 1,000,000$ [75]. Differential gene expression was calculated using Baggerley's test [76]. Fold change was calculated using $\mid \log 2$ fold change $\mid \geq 2$, and the $P$-value was set at $P<0.05$ [77].

\section{Novel miRNA prediction}

Novel miRNA prediction was carried out based on a previous study [78]. For secondary structure validation, forward and reverse primers were designed using the PrimerQuest Tool by Integrated DNA Technologies (https://sg.idtdna.com/), and the parameters were set to PCR 2 Primer (Table 6). The primer was synthesized at First Base (Malaysia). The PCR products were measured and visualized using $4 \%$ agarose gel.

\section{Prediction of target transcript and gene ontology}

PsRobot software was deployed to predict miRNA targets with a score of 4.0 to obtain more targets $[29,79]$. The miRNA libraries generated in this study and the transcriptomic library of $P$. minor (http://www.ncbi.nlm. nih.gov/sra/SRX669305) were used in this target prediction. Then, the target genes were classified through gene ontology analysis using WEGO software (http://wego. genomics.org.cn/) [80]. The target genes involved in the terpenoid pathway were simply determined by the gene annotations in the transcriptomic library [81]. The genes were mapped with the KEGG database (http://www.genome.jp/kegg/) to identify the involvement of these genes in the terpenoid pathway [82].

Table 6 List of primer for pre-miRNA validation of putative novel miRNA

\begin{tabular}{ll}
\hline Pre-miRNA & Sequence (5' to 3') \\
\hline Pre-nov_12 & 5'-CAC TाT CAC TCT CCT CCT CCA-3' (Forward) \\
& 5'-CCT CTT TCA CTT TCA CTT CCT CTT T-3' (Reverse) \\
Pre-nov_13 & 5'-AAG CCC TCC ATA TCA GCT CCT GAT-3' (Forward) \\
& 5'-CCC ATT CCT CCA CCA ACT CCT-3' (Reverse) \\
\hline
\end{tabular}

Table 7 List of miRNA primers for RT-qPCR

\begin{tabular}{ll}
\hline miRNA & Sequence (5' to 3') \\
\hline 5.8 s rRNA & 5'-ACG TCT GCC TGG GTG TCA CAA-3' (Forward) \\
Pmi-miR396a & 5'-GTT CAA GAA AGC TGT GGG A-3' (Forward) \\
Pmi-miR6173 & 5'-GGG GGA GCC GTA AAC GAT GGA TA-3' (Forward) \\
Pmi-miR398f/g & 5'-TGT GTC CTC AGG TCG CCC CCA-3' (Forward) \\
Pmi-miR530 & 5'-TAT CTG CAT TGT CAC CTG CAC CA-3' (Forward) \\
Pmi-miR6300 & 5'-GGG GGT GGT TGT AGT ATA GTG GA-3' (Forward) \\
Pmi-nov_12 & 5'-GAA AGA GGA AGT GAA AGT GAA-3' (Forward) \\
Pmi-nov_13 & 5'-GAG GAG TTG GTG GAG GAA-3' (Forward) \\
\hline
\end{tabular}

\section{Validation and expression profile using real-time quantitative PCR (RT-qPCR)}

To validate the existence and expression of those miRNAs and target genes involved in the terpenoid pathway under $F$. oxysporum inoculation, RT-qPCR was carried out using Maxima SYBR Green qPCR Master Mix (Thermo Fisher, USA) at a series of time points: 0 dpi, 3 dpi, $6 \mathrm{dpi}$, and $9 \mathrm{dpi}$. For miRNA, the miRNA sequence was used as the forward primer, and the universal primer

Table 8 List of target genes primer for RT-qPCR

\begin{tabular}{|c|c|}
\hline Target genes & Sequence $\left(5^{\prime}\right.$ to $\left.3^{\prime}\right)$ \\
\hline \multirow[t]{2}{*}{ Tubulin } & $\begin{array}{l}\text { 5'-TAC CAG CCA CCA ACC GTA GTC C-3' } \\
\text { (Forward) }\end{array}$ \\
\hline & $\begin{array}{l}\text { 5'-CCA ACC TCC TCG TAG TCT TTC TCA A-3' } \\
\text { (Reverse) }\end{array}$ \\
\hline \multirow[t]{2}{*}{ Peroxidase57 } & 5'-GGA ACC CAA ACC ACA ACT TTC-3' (Forward) \\
\hline & $\begin{array}{l}\text { 5'-CTG TCG CCA ATC TTT CAT CAA TC-3' } \\
\text { (Reverse) }\end{array}$ \\
\hline \multirow[t]{2}{*}{ DXS } & 5'-GGC GAA TाT GAA CTG GGT TG-3' (Forward) \\
\hline & 5'-GAT TTA GCT TGT GCT TGG ATG G-5 (Reverse) \\
\hline \multirow{2}{*}{$\begin{array}{l}\text { Sesquiterpene } \\
\text { synthase }\end{array}$} & 5'-AGA CGT AGT GAG CAA CCA AC-3' (Forward) \\
\hline & 5'-CTT GGC ATA CCC TTG TGG TAA-3' (Reverse) \\
\hline \multirow[t]{2}{*}{ FDS1 } & 5'-GGG ACG ATA CTT CTC GCA AT-3' (Forward) \\
\hline & 5'-GAG TGC ACT GGC TTG AAA GA-3' (Reverse) \\
\hline \multirow[t]{2}{*}{ DXR } & 5'-GAC GTT TAA AGC CCC AGA CA-3' (Forward) \\
\hline & 5'-AGG TCA GCT CAA CAA CCT TGA-3' (Reverse) \\
\hline \multirow[t]{2}{*}{ MVD } & $\begin{array}{l}\text { 5'-GCT TCA TTG AGA AAT GGA ACC G-3' } \\
\text { (Forward) }\end{array}$ \\
\hline & $\begin{array}{l}\text { 5'-AAC CTT CCT ATT ACG TGC GAT TA-3' } \\
\text { (Reverse) }\end{array}$ \\
\hline \multirow[t]{2}{*}{ HMGR } & 5'-GCC AAC ATT GTG TCT GCT ATC-3' (Forward) \\
\hline & 5'-ATG GTC ACG GAG ATG TGA AG-3' (Reverse) \\
\hline \multirow[t]{2}{*}{$\mathrm{ADH}$} & 5'-TTA GGC GGA AGA ACA CTC AAG-3' (Forward) \\
\hline & $\begin{array}{l}\text { 5'-CCA ACT TGA TCT CCT GGT TAA GA-3' } \\
\text { (Reverse) }\end{array}$ \\
\hline \multirow[t]{2}{*}{ MVK } & 5'-AAG GTA AAC GCT CCG ATT CC-3' (Forward) \\
\hline & $\begin{array}{l}\text { 5'-CAA TGC CGC GAG AAT TTG ATT A-3' } \\
\text { (Reverse) }\end{array}$ \\
\hline
\end{tabular}


from Qiagen was used as the reverse primer (Table 7). For the target genes, forward and reverse primers were designed using the PrimerQuest Tool by Integrated DNA Technologies (https://sg.idtdna.com/), and the parameters were set to PCR 2 Primer intercalating dye (Table 8). $5.8 \mathrm{~s} \mathrm{rRNA}$ and tubulin were used as reference genes for the miRNAs and target genes, respectively. Relative gene expression was calculated using the Livak method [83].

\section{Degradome sequencing}

To determine the miRNA cleavage site, RNA samples from $\mathrm{C}$ and $\mathrm{F}$ were pooled together and sent to $\mathrm{BGI}$ (China) for degradome sequencing. After retrieving the raw data, low-quality reads were discarded, and adaptors were removed using Skewer software [84]. Once again, psRobot was deployed to map the degradome sequence against the reference gene ( $P$. minor transcriptome) and to determine the cleavage site [29].

\section{Correlation analysis}

To obtain overall visualization of the relationship between miRNA metabolites and mRNA metabolites, non-parametric Spearman's rank correlation analysis was carried out using miRTarVis software (http://hcil. snu.ac.kr/ rati/miRTarVis/index.html) with the default settings [85].

\section{Additional files}

Additional file 1: SPME GC-MS data from the inoculation test. These data show the effect of $F$. oxysporum treatment on terpenoid content in P. minor plants at $0,3,6$, and 9 dpi. (CSV $1 \mathrm{~kb}$ )

Additional file 2: Measurement of RNA integrity for each sample. An RNA integrity check was carried out prior to small RNA library construction. (PPTX $148 \mathrm{~kb})$

Additional file 3: Spearman coefficients for miRNA-metabolite and mRNA-metabolite. Positive values indicate positive correlations, whereas negative values indicate negative correlations. (XLSX 8 kb)

\section{Abbreviations \\ ADH: Alcohol dehydrogenase; DXR: 1-deoxy-d-xylulose-5-phosphate reductoisomerase; DXS: 1-deoxy-d-xylulose-5-phosphate synthase: FDS: Farnesyl diphosphate synthase; GLV: Green leaf volatile; HMGR: 3- hydroxy-3-methylglutaryl-coenzyme A; MEP: Methylerythritol 4-phosphate; miRNA: MicroRNA; MVA: MevalonateMVDDiphosphomevalonate decarboxylase; MVK: Mevalonate kinase; nt: Nucleotide}

\section{Acknowledgements}

We would like to thank Centre for Research and Instrumentation (CRIM) Universiti Kebangsaan Malaysia for providing SPME GC-MS analysis. We also want to thank Malaysian Genome Institute (MGI) and Universiti Putra Malaysia (UPM) for their Bioanalyzer service.

\section{Authors' contributions}

AS designed and performed the laboratory work and interpreted the results. JJ carried out the bioinformatics and degradome analysis. AM and II advised the experimental design. RT and MN worked on the transcriptomic library. The first draft of this manuscript was written by AS and revised by MS and II.
The revised version were made by AS, MS and II. All authors read and approved the final manuscript.

\section{Funding}

The author AS is sponsored by MyBrain 15 (MyPhD) scholarship. This study on miRNA is funded by Dana Impak Perdana UKM (DIP-2015-018) from Universiti Kebangsaan Malaysia (UKM), Malaysia. The funding body provide us the space and lab facilities to carry out the experiments. Thus, allowed the data to be analyzed and interpreted by authors as well as preparing the manuscript. This also included recruiting the graduate student who perform the experimental work.

\section{Availability of data and materials}

The raw data of small RNA libraries were deposited in NCBI under accession number SRX2645686, SRX2645687, SRX2645684, SRX2645685. The transcriptome data can be access in NCBI under accession number SRX669305. The degradome sequencing data also were submitted to NCBI under accession number SRX3921398 and SRX3921610

Ethics approval and consent to participate

Not applicable.

\section{Consent for publication}

Not applicable.

\section{Competing interests}

The authors declare that they have no competing interests.

\section{Author details}

${ }^{1}$ School of Biosciences and Biotechnology, Faculty of Science and Technology, Universiti Kebangsaan Malaysia, UKM, 43600 Bangi, Selangor, Malaysia. ${ }^{2}$ Institute of Systems Biology, Universiti Kebangsaan Malaysia, UKM, 43600 Bangi, Selangor, Malaysia. ${ }^{3}$ Borneo Medical and Health Research Centre, Faculty of Medicine and Health Sciences, Universiti Malaysia Sabah, Kota Kinabalu, Malaysia. ${ }^{4}$ Department of Plant Breeding and Genetics, University College of Agriculture \& Environmental Sciences, The Islamia University of Bahawalpur, Punjab, Pakistan. ${ }^{5}$ Department of Biosciences, Faculty of Science, Universiti Teknologi Malaysia, 81310 Skudai, Johor, Malaysia.

Received: 9 July 2018 Accepted: 3 July 2019

Published online: 16 July 2019

\section{References}

1. Gupta OP, Karkute SG, Banerjee S, Meena NL, Dahuja A. Contemporary understanding of miRNA-based regulation of secondary metabolites biosynthesis in plants. Front Plant Sci. 2017;8(374).

2. Mazid M, Khan TA, Mohammad F. Role of secondary metabolites in defense mechanisms of plants. Biol Med. 2011;3(2):232-49.

3. Dubey VS, Bhalla R, Luthra R. An overview of the non-mevalonate pathway for terpenoid biosynthesis in plants. J Biosci. 2003;28(5):637-46.

4. Vikram P, Chiruvella KK, Ripain IHA, Arifullah M. A recent review on phytochemical constituents and medicinal properties of kesum (Polygonum minus Huds.). Asian Pac J Trop Biomed. 2014;4(6):430-5.

5. Abbas F, Ke Y, Yu R, Yue Y, Amanullah S, Jahangir MM, Fan Y. Volatile terpenoids: multiple functions, biosynthesis, modulation and manipulation by genetic engineering. Planta. 2017;246(5):803-16.

6. Ahmad R, Baharum SN, Bunawan H, Lee M, Mohd Noor N, Rohani ER, llias N Zin NM. Volatile profiling of aromatic traditional medicinal plant, Polygonum minus in different tissues and its biological activities. Molecules. 2014;19(11): 19220-42.

7. Christapher PV, Parasuraman S, Christina JM, Asmawi MZ, Vikneswaran M. Review on Polygonum minus. Huds, a commonly used food additive in Southeast Asia. Pharm Res. 2015;7(1):1-6.

8. Baharum SN, Bunawan H, Ghani MA, Mustapha WA, Noor NM. Analysis of the chemical composition of the essential oil of Polygonum minus Huds. using two-dimensional gas chromatography-time-of-flight mass spectrometry (GC-TOF MS). Molecules. 2010;15(10):7006-15.

9. Nagegowda DA. Plant volatile terpenoid metabolism: biosynthetic genes, transcriptional regulation and subcellular compartmentation. FEBS Lett. 2010;584(14):2965-73. 
10. Tholl D. Biosynthesis and biological functions of terpenoids in plants. Adv Biochem Eng Biotechnol. 2015;148:63-106

11. AFA S, Sajad M, Nazaruddin N, Fauzi IA, AMA M, Zainal Z, Ismail I. MicroRNA and transcription factor: key players in plant regulatory network. Front Plant Sci. 2017;8(565).

12. Ramakrishna A, Ravishankar GA. Influence of abiotic stress signals on secondary metabolites in plants. Plant Signal Behav. 2011;6(11):1720-31.

13. Ramirez-Estrada K, Vidal-Limon H, Hidalgo D, Moyano E, Golenioswki M, Cusido RM, Palazon J. Elicitation, an effective strategy for the biotechnological production of bioactive high-added value compounds in plant cell factories. Molecules. 2016;21(2):182.

14. Ee S-F, Mohamed-Hussein Z-A, Othman R, Shaharuddin NA, Ismail I, Zainal Z. Functional characterization of Sesquiterpene synthase from Polygonum minus. Sci World J. 2014;2014:840592.

15. Zhao J, Davis LC, Verpoorte R. Elicitor signal transduction leading to production of plant secondary metabolites. Biotechnol Adv. 2005;23(4):283-333.

16. Gadzovska Simic S, Tusevski O, Maury S, Hano C, Delaunay A, Chabbert B, Lamblin F, Lainé E, Joseph C, Hagège D. Fungal elicitor-mediated enhancement in phenylpropanoid and naphtodianthrone contents of Hypericum perforatum $\mathrm{L}$. cell cultures. Plant Cell Tissue Organ Cult. 2015;122(1):213-26.

17. Pereira PS, Ticli FK, SdC F, CMdS B, Lourenço MV. Enhanced triterpene production in Tabernaemontana catharinensis cell suspension cultures in response to biotic elicitors. Quím Nova. 2007;30:1849-52.

18. Silvar C, Merino F, Diaz J. Resistance in pepper plants induced by Fusarium oxysporum f. sp. lycopersici involves different defence-related genes. Plant Biol (Stuttg). 2009;11(1):68-74.

19. Naik PM, Al-Khayri JM. Abiotic and Biotic Elicitors-Role in Secondary Metabolites Production through In Vitro Culture of Medicinal Plants. In: Shanker AK, Rijeka SC, editors. Abiotic and Biotic Stress in Plants - Recent Advances and Future Perspectives; 2016. p. Ch. 10. InTech.

20. Jones-Rhoades M, Bartel D, Bartel B. MicroRNAS and their regulatory roles in plants. Annu Rev Plant Biol. 2006:57:19-53.

21. Motameny S, Wolters S, Nurnberg P, Schumacher B. Next generation sequencing of miRNAs - strategies, Resources and Methods. Genes. 2010;1(1):70-84.

22. Samad AFA, Ali NM, Ismail I. ILLUMINA ${ }^{\oplus}$ TRUSEQ $^{\circledR}$ VS NEBNEXT ${ }^{\circledR}$ SMALL RNA LIBRARY PREPARATION KIT FOR MIRNA PROFILING IN PERSICARIA MINOR: WHICH BETTER? Jurnal Teknologi. 2015;77(24):131-4.

23. Kozomara A, Griffiths-Jones S. miRBase: annotating high confidence microRNAs using deep sequencing data. Nucleic Acids Res. 2014;42:D68-73.

24. Piasecka A, Jedrzejczak-Rey N, Bednarek P. Secondary metabolites in plant innate immunity: conserved function of divergent chemicals. New Phytol. 2015;206(3):948-64.

25. Xuan P, Guo M, Huang Y, Li W, Huang Y. MaturePred: efficient identification of microRNAs within novel plant pre-miRNAs. PLoS One. 2011;6(11):e27422.

26. Loke K-K, Rahnamaie-Tajadod R, Yeoh C-C, Goh H-H, Mohamed-Hussein Z-A, Mohd Noor N, Zainal Z, Ismail I. RNA-seq analysis for secondary metabolite pathway gene discovery in Polygonum minus. Genom Data. 2016;7:12-3.

27. Stadhouders R, Pas SD, Anber J, Voermans J, Mes THM, Schutten M. The effect of primer-template mismatches on the detection and quantification of nucleic acids using the 5' nuclease assay. J Mol Diagn. 2010;12(1):109-17.

28. Bartel D. MicroRNAs: genomics, biogenesis, mechanism, and function. Cell. 2004;116:281-97.

29. Wu HJ, Ma YK, Chen T, Wang M, Wang XJ. PsRobot: a web-based plant small RNA meta-analysis toolbox. Nucleic Acids Res. 2012;40(Web Server issue):W22-8.

30. War AR, Paulraj MG, Ahmad T, Buhroo AA, Hussain B, Ignacimuthu S, Sharma HC. Mechanisms of plant defense against insect herbivores. Plant Signal Behav. 2012;7(10):1306-20.

31. Niinemets Ü, Kännaste A, Copolovici L. Quantitative patterns between plant volatile emissions induced by biotic stresses and the degree of damage. Front Plant Sci. 2013:4:262

32. Gor M, Ismail I, Mustapha W, Zainal Z, Noor N, Othman R, Hussein Z. Identification of cDNAs for jasmonic acid-responsive genes in Polygonum minus roots by suppression subtractive hybridization. Acta Physiol Plant. 2011;33(2):283-94.

33. Mercke P, Kappers IF, Verstappen FW, Vorst O, Dicke M, Bouwmeester HJ. Combined transcript and metabolite analysis reveals genes involved in spider mite induced volatile formation in cucumber plants. Plant Physiol. 2004;135(4):2012-24

34. Liu D, Song Y, Chen Z, Yu D. Ectopic expression of miR396 suppresses GRF target gene expression and alters leaf growth in Arabidopsis. Physiol Plant. 2009;136(2):223-36.
35. Baucher M, Moussawi J, Vandeputte OM, Monteyne D, Mol A, Perez-Morga D, El Jaziri M. A role for the miR396/GRF network in specification of organ type during flower development, as supported by ectopic expression of Populus trichocarpa miR396c in transgenic tobacco. Plant Biol (Stuttg). 2013; 15(5):892-8.

36. Soto-Suárez M, Baldrich P, Weigel D, Rubio-Somoza I, San Segundo B. The Arabidopsis miR396 mediates pathogen-associated molecular patterntriggered immune responses against fungal pathogens. Sci Rep. 2017;7: 44898.

37. Gao F, Wang K, Liu Y, Chen Y, Chen P, Shi Z, Luo J, Jiang D, Fan F, Zhu Y, et al. Blocking miR396 increases rice yield by shaping inflorescence architecture. Nat Plants. 2015;2:15196.

38. Jagadeeswaran G, Saini A, Sunkar R. Biotic and abiotic stress down-regulate miR398 expression in Arabidopsis. Planta. 2009;229(4):1009-14.

39. Sunkar R, Li Y-F, Jagadeeswaran G. Functions of microRNAs in plant stress responses. Trends Plant Sci. 2012;17:196-203.

40. Zhu C, Ding Y, Liu H. MiR398 and plant stress responses. Physiol Plant. 2011; 143(1):1-9.

41. Trindade I, Capitao C, Dalmay T, Fevereiro MP, Santos DM. miR398 and miR408 are up-regulated in response to water deficit in Medicago truncatula. Planta. 2010;231(3):705-16.

42. Hackenberg M, Gustafson P, Langridge P, Shi B-J. Differential expression of microRNAs and other small RNAs in barley between water and drought conditions. Plant Biotechnol J. 2015;13(1):2-13.

43. Srivastava S, Zheng Y, Kudapa H, Jagadeeswaran G, Hivrale V, Varshney RK, Sunkar R. High throughput sequencing of small RNA component of leaves and inflorescence revealed conserved and novel miRNAs as well as phasiRNA loci in chickpea. Plant Sci. 2015;235:46-57.

44. Kohli D, Joshi G, Deokar AA, Bhardwaj AR, Agarwal M, Katiyar-Agarwal S, Srinivasan R, Jain PK. Identification and characterization of wilt and salt stress-responsive MicroRNAs in chickpea through high-throughput sequencing. PLoS One. 2014;9(10):e108851.

45. Han X, Yin H, Song X, Zhang Y, Liu M, Sang J, Jiang J, Li J, Zhuo R. Integration of small RNAs, degradome and transcriptome sequencing in hyperaccumulator Sedum alfredii uncovers a complex regulatory network and provides insights into cadmium phytoremediation. Plant Biotechnol J. 2016;14(6):1470-83.

46. Li Y, Wan L, Bi S, Wan X, Li Z, Cao J, Tong Z, Xu H, He F, Li X. Identification of drought-responsive MicroRNAs from roots and leaves of alfalfa by highthroughput sequencing. Genes. 2017:8(4):119.

47. UI Hassan MN, Zainal Z, Ismail I. Green leaf volatiles: biosynthesis, biological functions and their applications in biotechnology. Plant Biotechnol J. 2015; 13(6):727-39.

48. Zhang B, Pan X, Cobb GP, Anderson TA. Plant microRNA: a small regulatory molecule with big impact. Dev Biol. 2006;289(1):3-16.

49. Aukerman $M$, Sakai H. Regulation of flowering time and floral organ identity by a MicroRNA and its APETALA2-like target genes. Plant Cell. 2003;15:2730-41.

50. Fan R, Li Y, Li C, Zhang Y. Differential microRNA analysis of glandular trichomes and young leaves in Xanthium strumarium L. Reveals their putative roles in regulating terpenoid biosynthesis. PLoS One. 2015;10(9): e0139002.

51. Enjuto M, Lumbreras V, Marin C, Boronat A. Expression of the Arabidopsis HMG2 gene, encoding 3-hydroxy-3-methylglutaryl coenzyme a reductase, is restricted to meristematic and floral tissues. Plant Cell. 1995;7(5):517-27.

52. Tholl D, Lee S. Terpene specialized metabolism in Arabidopsis thaliana. Arabidopsis Book. 2011;9:e0143.

53. Kondo K, Uritani I, Oba K. Induction mechanism of 3-hydroxy-3methylglutaryl-CoA reductase in potato tuber and sweet potato root tissues. Biosci Biotechnol Biochem. 2003;67(5):1007-17.

54. Suzuki M, Kamide Y, Nagata N, Seki H, Ohyama K, Kato H, Masuda K, Sato S, Kato T, Tabata S, et al. Loss of function of 3-hydroxy-3-methylglutaryl coenzyme a reductase 1 (HMG1) in Arabidopsis leads to dwarfing, early senescence and male sterility, and reduced sterol levels. Plant J. 2004;37(5):750-61.

55. Ohyama K, Suzuki M, Masuda K, Yoshida S, Muranaka T. Chemical phenotypes of the hmg1 and hmg2 mutants of Arabidopsis demonstrate the in-planta role of HMG-CoA reductase in triterpene biosynthesis. Chem Pharm Bull. 2007;55(10):1518-21.

56. Chappell J, VonLanken C, Vögeli U. Elicitor-inducible 3-Hydroxy-3Methylglutaryl coenzyme a reductase activity is required for Sesquiterpene accumulation in tobacco cell suspension cultures. Plant Physiol. 1991:97(2): 693-8. 
57. Krutzfeldt J, Rajewsky N, Braich R, Rajeev KG, Tuschl T, Manoharan M, Stoffel M. Silencing of microRNAs in vivo with 'antagomirs'. Nature. 2005;438(7068):685-9.

58. Michihara A, Ide N, Mizutani Y, Okamoto M, Uchida M, Matsuoka H, Akasaki $\mathrm{K}$. Involvement of microRNA214 and transcriptional regulation in reductions in mevalonate pyrophosphate decarboxylase mRNA levels in stroke-prone spontaneously hypertensive rat livers. Biosci Biotechnol Biochem. 2015; 79(11):1759-70.

59. Singh B, Sharma RA. Plant terpenes: defense responses, phylogenetic analysis, regulation and clinical applications. 3 Biotech. 2015;5(2):129-51.

60. Chen Q, Yan J, Meng X, Xu F, Zhang W, Liao Y, Qu J. Molecular cloning, characterization, and functional analysis of acetyl-CoA C-acetyltransferase and mevalonate kinase genes involved in terpene trilactone biosynthesis from Ginkgo biloba. Molecules. 2017;22(1):74.

61. Estevez JM, Cantero A, Reindl A, Reichler S, Leon P. 1-deoxy-D-xylulose-5phosphate synthase, a limiting enzyme for plastidic isoprenoid biosynthesis in plants. J Biol Chem. 2001;276(25):22901-9.

62. Mandel MA, Feldmann KA, Herrera-Estrella L, Rocha-Sosa M, Leon P. CLA1, a novel gene required for chloroplast development, is highly conserved in evolution. Plant J. 1996;9(5):649-58

63. Phillips MA, Leon P, Boronat A, Rodriguez-Concepcion M. The plastidial MEP pathway: unified nomenclature and resources. Trends Plant Sci. 2008;13(12): 619-23

64. Paetzold H, Garms S, Bartram S, Wieczorek J, Uros-Gracia EM, RodriguezConcepcion M, Boland W, Strack D, Hause B, Walter MH. The isogene 1deoxy-D-xylulose 5-phosphate synthase 2 controls isoprenoid profiles, precursor pathway allocation, and density of tomato trichomes. Mol Plant. 2010;3(5):904-16

65. Carretero-Paulet L, Galstyan A, Roig-Villanova I, Martinez-Garcia JF, BilbaoCastro JR, Robertson DL. Genome-wide classification and evolutionary analysis of the bHLH family of transcription factors in arabidopsis, poplar, rice, moss, and algae. Plant Physiol. 2010;153:1398-412.

66. Xing S, Miao J, Li S, Qin G, Tang S, Li H, Gu H, Qu LJ. Disruption of the 1deoxy-D-xylulose-5-phosphate reductoisomerase (DXR) gene results in albino, dwarf and defects in trichome initiation and stomata closure in Arabidopsis. Cell Res. 2010;20(6):688-700.

67. Yu ZX, Wang LJ, Zhao B, Shan CM, Zhang YH, Chen DF, Chen XY. Progressive regulation of Sesquiterpene biosynthesis in Arabidopsis and patchouli (Pogostemon cablin) by the miR156-targeted SPL transcription factors. Mol Plant. 2014;8:98-110.

68. Hong $Y$, Jackson S. Floral induction and flower formation - the role and potential applications of miRNAs. Plant Biotechnol J. 2015;13(3):282-92.

69. Samad AFA, Nazaruddin N, Sajad M, Jani J, Murad AMA, Zainal Z, Ismail I. Small RNA sequencing for secondary metabolite analysis in Persicaria minor. Genom Data. 2017;13:3-4.

70. Câmara JS, Arminda Alves M, Marques JC. Development of headspace solid-phase microextraction-gas chromatography-mass spectrometry methodology for analysis of terpenoids in Madeira wines. Analytica Chimica Acta 2006;555(2):191-200.

71. Xia J, Wishart DS. Using MetaboAnalyst 3.0 for comprehensive metabolomics data analysis. Curr Protoc Bioinformatics. 2016:55:14.10.11-91.

72. Accerbi M, Schmidt SA, De Paoli E, Park S, Jeong DH, Green PJ. Methods for isolation of total RNA to recover miRNAs and other small RNAs from diverse species. Methods Mol Biol. 2010;592:31-50.

73. Nawrocki EP, Burge SW, Bateman A, Daub J, Eberhardt RY, Eddy SR, Floden EW, Gardner PP, Jones TA, Tate J, et al. Rfam 12.0: updates to the RNA families database. Nucleic Acids Res. 2015;43(D1):D130-7.

74. Yang L, Jue D, Li W, Zhang R, Chen M, Yang Q. Identification of MiRNA from eggplant (Solanum melongena L.) by small RNA deep sequencing and their response to Verticillium dahliae infection. PLoS One. 2013;8(8):e72840.

75. Wang Y, Li L, Tang S, Liu J, Zhang H, Zhi H, Jia G, Diao X. Combined small RNA and degradome sequencing to identify miRNAs and their targets in response to drought in foxtail millet. BMC Genet. 2016;17:57.

76. Baggerly KA, Deng L, Morris JS, Aldaz CM. Differential expression in SAGE: accounting for normal between-library variation. Bioinformatics. 2003;19(12):1477-83.

77. Audic S, Claverie JM. The significance of digital gene expression profiles. Genome Res. 1997;7:986-95.

78. AFA S, Nazaruddin N, AMA M, Jani J, Zainal Z, Ismail I. Deep sequencing and in silico analysis of small RNA library reveals novel miRNA from leaf Persicaria minor transcriptome. 3 Biotech. 2018;8(3):136.

79. Yuan J, Zhang Y, Dong J, Sun Y, Lim BL, Liu D, Lu ZJ. Systematic characterization of novel IncRNAs responding to phosphate starvation in Arabidopsis thaliana. BMC Genomics. 2016;17(1):655.
80. Ye J, Fang L, Zheng H, Zhang Y, Chen J, Zhang Z, Wang J, Li S, Li R, Bolund L, et al. WEGO: a web tool for plotting GO annotations. Nucleic Acids Res. 2006;34(suppl_2):W293-7.

81. Rahnamaie-Tajadod R, Loke K-K, Goh H-H, Mohd Noor N. Differential gene expression analysis in Polygonum minus leaf upon 24 hours of methyl jasmonate elicitation. Front Plant Sci. 2017;8:109.

82. Kanehisa M, Furumichi M, Tanabe M, Sato Y, Morishima K. KEGG: new perspectives on genomes, pathways, diseases and drugs. Nucleic Acids Res. 2017:45(D1):D353-61.

83. Livak KJ, Schmittgen TD. Analysis of relative gene expression data using real-time quantitative PCR and the 2(-Delta Delta C (T)) method. Methods. 2001;25:402-8

84. Jiang H, Lei R, Ding S-W, Zhu S. Skewer: a fast and accurate adapter trimmer for next-generation sequencing paired-end reads. BMC Bioinformatics. 2014; 15(1):182.

85. Jung D, Kim B, Freishtat RJ, Giri M, Hoffman E, Seo J. miRTarVis: an interactive visual analysis tool for microRNA-mRNA expression profile data. BMC Proc. 2015;9(Suppl 6 Proceedings of the 5th Symposium on Biological Data):S2.

\section{Publisher's Note}

Springer Nature remains neutral with regard to jurisdictional claims in published maps and institutional affiliations.

\section{Ready to submit your research? Choose BMC and benefit from:}

- fast, convenient online submission

- thorough peer review by experienced researchers in your field

- rapid publication on acceptance

- support for research data, including large and complex data types

- gold Open Access which fosters wider collaboration and increased citations

- maximum visibility for your research: over $100 \mathrm{M}$ website views per year

At BMC, research is always in progress.

Learn more biomedcentral.com/submissions 